\title{
Efficient Synthesis and Host-guest Properties of a New
}

\section{Class of Calix[6]aza-cryptands}

\author{
Stéphane Le Gac, Xianshun Zeng, Camille Girardot, Ivan Jabin*
}

\section{Supporting Information}

\section{List of content}

\section{Experimental Section SI2}

Figure S1. ${ }^{13} \mathrm{C}$ NMR spectrum $\left(75 \mathrm{MHz}, \mathrm{CDCl}_{3}\right)$ of compound 2. SI4

Figure S2. ${ }^{1} \mathrm{H}$ NMR spectrum $\left(300 \mathrm{MHz}, \mathrm{CDCl}_{3}\right.$ ) of compound 3. SI4

Figure S3. ${ }^{13} \mathrm{C} \mathrm{NMR}$ spectrum $\left(75 \mathrm{MHz}, \mathrm{CDCl}_{3}\right)$ of compound 3. SI5

Figure S4. ${ }^{1} \mathrm{H}$ NMR spectrum $\left(300 \mathrm{MHz}, \mathrm{CDCl}_{3}\right)$ of crude compound 5. SI5

Figure S5. ${ }^{13} \mathrm{C}$ NMR spectrum $\left(75 \mathrm{MHz}, \mathrm{CDCl}_{3}\right.$ ) of calix[6]tampo 6. SI6

Figure S6. HMBC spectrum $\left(\mathrm{CDCl}_{3}\right)$ of calix[6]tampo 6. SI6

Figure S7. HMQC spectrum $\left(\mathrm{CDCl}_{3}\right)$ of calix[6]tampo 6. SI7

Figure S8. COSY spectrum $\left(\mathrm{CDCl}_{3}\right)$ of calix[6] tampo 6. SI7

Figure S9. ${ }^{1} \mathrm{H}$ NMR spectra $(300 \mathrm{MHz})$ of protected calix[6]tampo 7. a) in $\mathrm{CDCl}_{3}$ at $293 \mathrm{~K}$; b) in toluene-d8 at $353 \mathrm{~K}$. SI8

Figure S10. HMQC spectrum $\left(\mathrm{CDCl}_{3}\right)$ of protected calix[6] tampo 7. SI8

Figure S11. COSY spectrum $\left(\mathrm{CDCl}_{3}\right)$ of protected calix[6]tampo 7. SI9

Figure S12. ${ }^{1} \mathrm{H}$ NMR spectrum $\left(300 \mathrm{MHz}, \mathrm{CDCl}_{3}\right.$ ) of crude compound 8. SI9

Figure S13. ${ }^{13} \mathrm{C}$ NMR spectrum $\left(75 \mathrm{MHz}, \mathrm{CDCl}_{3}\right.$ ) of crude compound 8. SI10

Figure S14. HMQC spectrum $\left(\mathrm{CDCl}_{3}\right)$ of crude compound 8. SI10

Figure S15. ${ }^{13} \mathrm{C}$ NMR spectrum $\left(75 \mathrm{MHz}, \mathrm{CDCl}_{3}\right)$ of calix[6]tamb 9. SI1 1

Figure S16. HMBC spectrum $\left(\mathrm{CDCl}_{3}\right)$ of calix[6]tamb 9. SI1 1

Figure S17. HMQC spectrum $\left(\mathrm{CDCl}_{3}\right)$ of calix[6]tamb 9. SI12

Figure S18. COSY spectrum $\left(\mathrm{CDCl}_{3}\right)$ of calix[6]tamb 9. SI12

Figure S19. NOESY spectrum $\left(\mathrm{CDCl}_{3}\right)$ of the host-guest complex $\mathbf{9 . 3 \mathbf { H } ^ { + }} \supset \mathbf{I M I}$. SI13

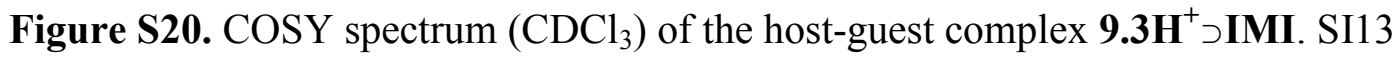

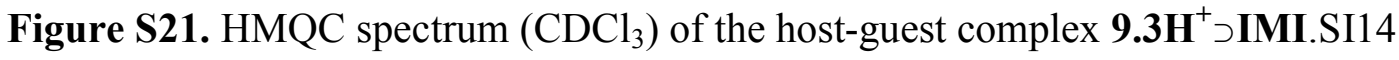

Figure S22. HMBC spectrum $\left(\mathrm{CDCl}_{3}\right)$ of the host-guest complex $\mathbf{9 . 3 \mathbf { H } ^ { + }} \supset$ IMI. SI14

Figure S23. ${ }^{1} \mathrm{H}$ NMR spectra $\left(300 \mathrm{MHz}, \mathrm{CDCl}_{3}\right)$ of a) calix[6]tamb 9; b) fully protonated calix[6] tamb $\mathbf{9 . 3 \mathbf { H } ^ { + }}$ c) host-guest complex $\mathbf{9 . 3 \mathbf { H } ^ { + }} \supset \mathbf{I M I}$. SI15 


\section{General methods}

All the reactions were performed under an inert atmosphere. $\mathrm{CH}_{2} \mathrm{Cl}_{2}$ was distilled over $\mathrm{CaH}_{2}$ under argon. Ethanol was distilled over sodium/diethylphtalate under argon. Silica gel (230-400 mesh) was used for flash chromatography separations. ${ }^{1} \mathrm{H}$ and ${ }^{13} \mathrm{C}$ NMR spectra were recorded respectively at $300 \mathrm{MHz}$ and $75 \mathrm{MHz}$. Chemical shifts are expressed in ppm. Traces of residual solvent or poly(dimethylsiloxane) (R) were used as internal standard. All the signals of ${ }^{1} \mathrm{H}$ NMR spectra were attributed through 2D NMR analyses (COSY, HMQC, HMBC).

\section{Procedure for the determination of the relative affinities of the neutral molecules toward host} 9.3H ${ }^{+}$through ${ }^{1}$ H NMR competitive binding studies: TFA (6 équiv.), PYD (16 equiv.) and IMI (3 equiv.) were successively added into a $\mathrm{CDCl}_{3}$ solution $(0.60 \mathrm{~mL})$ containing calix[6] tamb $9(3 \mathrm{mg}$, $1.96 \mu \mathrm{mol})$. A ${ }^{1} \mathrm{H}$ NMR spectrum recorded at $\mathrm{rt}$ showed the resonances of both endo-complexes 9.3H ${ }^{+} \supset \mathbf{I M I}$ and $9.3 \mathbf{H}^{+} \supset \mathbf{P Y D}$ besides the signals corresponding to $\mathrm{PYD}_{\text {free }}$ and $\mathrm{IMI}_{\text {free. Integration of }}$ the signals of the included guests, ie. $\mathrm{IMI}_{\text {in }}$ and $\mathrm{PYD}_{\text {in }}$, afforded a 6.5:1 ratio for $\mathrm{IMI}_{\text {in }} / \mathrm{PYD}_{\text {in }}$. Thus, the relative affinity, defined as $\left[\mathrm{IMI}_{\text {in }}\right] /\left[\mathrm{PYD}_{\text {in }}\right] \times\left[\mathrm{PYD}_{\text {free }}\right] /\left[\mathrm{IMI}_{\text {free }}\right]$, was found to be 0.02 (errors estimated $\pm 10 \%$ ). Ratio of neutral molecules used for the other NMR competitive binding experiment: $\mathrm{IMI} / \mathrm{DMSO}=36$.

Complex 9.3H $\mathbf{H}^{+}$IMI. Calix[6]tamb 9 (3 mg, $\left.1.96 \mu \mathrm{mol}\right)$ was dissolved in $\mathrm{CDCl}_{3}(0.6 \mathrm{~mL})$ previously filtered through basic alumina. TFA (6 equiv.) and IMI (24 equiv.) were then successively added and a ${ }^{1} \mathrm{H}$ NMR spectrum was recorded showing host-guest complex $9.3 \mathbf{H}^{+} \supset \mathbf{I M I}$ as the unique species.

1,3,5-Tri(2'-formylbenzyl)benzene 3: 1,3,5-tris(bromomethyl)benzene 1 (2.20 g, $6.16 \mathrm{mmol}$ ) and 2-formylphenylboronic acid (3.05 g, $20.35 \mathrm{mmol})$ were dissolved in a deoxygenated mixture of toluene $(185 \mathrm{~mL})$ and aqueous $\mathrm{Na}_{2} \mathrm{CO}_{3}$ solution $(1 \mathrm{M}, 185 \mathrm{ml}) . \mathrm{Pd}\left(\mathrm{PPh}_{3}\right)_{4}(855 \mathrm{mg}, 0.74 \mathrm{mmol})$ was added and the reaction mixture was heated at $90^{\circ} \mathrm{C}$ for 2 days. The organic layer was separated, washed with water, dried with $\mathrm{MgSO}_{4}$ and filtered. After concentration, the resulting residue was purified by column chromatography $\left(\mathrm{CH}_{2} \mathrm{Cl}_{2} / \mathrm{Et}_{3} \mathrm{~N}, 99.5: 0.5\right)$ yielding compound 3 (1.166 g, $44 \%$ ) as a pale yellow solid. mp $110{ }^{\circ} \mathrm{C}$. IR (KBr): v $1694 \mathrm{~cm}^{-1}$. NMR ${ }^{1} \mathrm{H}\left(300 \mathrm{MHz}, \mathrm{CDCl}_{3}\right) \delta 4.32(\mathrm{~s}$, $\left.6 \mathrm{H}, \mathrm{ArCH}_{2}\right), 6.73$ (s, 3H, ArH), 7.15 (d, $\left.J=8 \mathrm{~Hz}, 3 \mathrm{H}, \mathrm{ArH}\right), 7.39$ (t, $\left.J=8 \mathrm{~Hz}, 3 \mathrm{H}, \mathrm{ArH}\right), 7.49$ (t, $J=$ $8 \mathrm{~Hz}, 3 \mathrm{H}, \mathrm{ArH}), 7.82$ (d, $J=8 \mathrm{~Hz}, 3 \mathrm{H}, \mathrm{ArH}), 10.16$ (s, 3H, CHO). NMR ${ }^{13} \mathrm{C}\left(75 \mathrm{MHz}, \mathrm{CDCl}_{3}\right) \delta$ $40.0\left(\mathrm{CH}_{2}\right), 127.1(\mathrm{CH}), 127.5(\mathrm{CH}), 131.6(\mathrm{CH}), 132.2(\mathrm{CH}), 134.0(\mathrm{C}), 134.1(\mathrm{CH}), 141.0(\mathrm{C})$, 142.9 (C), $192.5(\mathrm{CH})$. Anal. Calcd for $\mathrm{C}_{30} \mathrm{H}_{24} \mathrm{O}_{3} .0 .5 \mathrm{H}_{2} \mathrm{O}: \mathrm{C}, 81.61 ; \mathrm{H}, 5.71$. Found: C, 81.89; H, 5.61 .

Calix[6]tris-amine 4. Calix[6] tris-ester ${ }^{12}(0.595 \mathrm{~g}, 0.47 \mathrm{mmol})$ and anhydrous methanol $(25 \mathrm{~mL})$ were introduced in a reactor and a strong ammoniac bubbling was performed over $30 \mathrm{~min}$. The reactor was sealed and the reaction mixture was heated at $55^{\circ} \mathrm{C}$ for $16 \mathrm{~h}$. After cooling to rt, the methanol was removed under reduced pressure affording pure calix[6]tris-amide $(0.537 \mathrm{~g}, 97 \%)$ as a white solid. The subsequent reduction of this calix[6]tris-amide by $\mathrm{BH}_{3} / \mathrm{THF}$ was performed in $85 \%$ yield according to the literature ${ }^{4 \mathrm{~b}}$ affording pure calix[6]tris-amine 4 (79\% overall yield from $\left.\mathrm{X}_{6} \mathrm{H}_{3} \mathrm{Me}_{3}\right)$ 
Protected calix[6]tampo 7: mp 173-175 ${ }^{\circ} \mathrm{C} . \mathrm{IR}(\mathrm{KBr}): v 1696 \mathrm{~cm}^{-1} .{ }^{1} \mathrm{H}$ NMR $\left(300 \mathrm{MHz}, \mathrm{CDCl}_{3}\right) \delta$ $0.75\left(\mathrm{~s}, 27 \mathrm{H}, t \mathrm{Bu}_{\text {calix }}\right), 1.38\left(\mathrm{~s}, 45 \mathrm{H}, t \mathrm{Bu}_{\text {calix }}+2 t \mathrm{Bu}_{\mathrm{Boc}}\right), 1.46\left(\mathrm{~s}, 9 \mathrm{H}, t \mathrm{Bu}_{\mathrm{Boc}}\right), 2.07-2.20(\mathrm{~m}, 9 \mathrm{H}, \mathrm{OMe})$, 3.20-3.38 (m, 6H, $\left.\mathrm{ArCH}_{e q}\right), 3.72-3.82\left(\mathrm{~m}, 6 \mathrm{H}, \mathrm{OCH}_{2} \mathrm{CH}_{2} \mathrm{~N}\right), 3.85-3.95\left(\mathrm{~m}, 2 \mathrm{H}, \mathrm{OCH}_{2} \mathrm{CH}_{2} \mathrm{~N}\right), 3.96-$ $4.04\left(\mathrm{~m}, 4 \mathrm{H}, \mathrm{OCH}_{2} \mathrm{CH}_{2} \mathrm{~N}\right), 4.38-4.60\left(\mathrm{~m}, 6 \mathrm{H}, \mathrm{ArCH}_{a x}\right), 4.78-4.94\left(\mathrm{~m}, 6 \mathrm{H}, \mathrm{ArCH}_{2} \mathrm{~N}\right), 5.22-5.35(\mathrm{~m}$, 6H, $\left.\mathrm{ArCH}_{2} \mathrm{O}\right), 6.51-6.62$ (m, 6H, ArH calix), 6.79-6.89 (m, 3H, $\mathrm{ArH}_{\text {cap }}$ ), 6.90-6.98 (m, 3H, ArH cap), 7.06-7.38 (m, 12H, $\left.\mathrm{ArH}_{\text {cap }}+\mathrm{ArH}_{\text {calix }}\right), \quad 7.54-7.67\left(\mathrm{~m}, 3 \mathrm{H}, \quad \mathrm{ArH}_{\text {cap }}\right) . \quad$ Anal. Calcd for $\mathrm{C}_{120} \mathrm{H}_{153} \mathrm{~N}_{3} \mathrm{O}_{15} . \mathrm{H}_{2} \mathrm{O}$ : C, 76.04; H, 8.24; N, 2.22. Found: C, 75.77; H, 8.13; N, 2.22.

Calix[6]tampo 6: $\mathrm{mp} 265{ }^{\circ} \mathrm{C}(\mathrm{dec})$. IR (KBr): $v 3700$ to $3120,2960,1483,1454,1239 \mathrm{~cm}^{-1} .{ }^{1} \mathrm{H}$ NMR $\left(300 \mathrm{MHz}, \mathrm{CDCl}_{3}\right) \delta 0.71(\mathrm{~s}, 27 \mathrm{H}, t \mathrm{Bu}), 1.37(\mathrm{~s}, 27 \mathrm{H}, t \mathrm{Bu}), 1.61(\mathrm{~s}, 9 \mathrm{H}, \mathrm{OMe}), 3.15(\mathrm{~s}, 6 \mathrm{H}$, $\left.\mathrm{OCH}_{2} \mathrm{CH}_{2} \mathrm{~N}\right), 3.30\left(\mathrm{~d}, J=15 \mathrm{~Hz}, 6 \mathrm{H}, \mathrm{ArCH}_{e q}\right), 4.11\left(\mathrm{~s}, 12 \mathrm{H}, \mathrm{OCH}_{2} \mathrm{CH}_{2} \mathrm{~N}+\mathrm{ArCH} H_{2} \mathrm{~N}\right), 4.52(\mathrm{~d}, J=15$ $\left.\mathrm{Hz}, 6 \mathrm{H}, \mathrm{ArCH}_{a x}\right), 5.54\left(\mathrm{~s}, 6 \mathrm{H}, \mathrm{ArCH}_{2} \mathrm{O}\right), 6.53$ (s, 6H, $\left.\mathrm{ArH}_{\text {calix }}\right), 6.88$ (t, J=7 Hz, 3H, ArH $\mathrm{Aap}_{\text {cap }}, 6.89$ (d, $\left.J=8 \mathrm{~Hz}, 3 \mathrm{H}, \mathrm{ArH}_{\text {cap }}\right), 7.13$ (t, $\left.J=8 \mathrm{~Hz}, 3 \mathrm{H}, \mathrm{ArH}_{\text {cap }}\right), 7.19$ (s, 6H, $\left.\operatorname{ArH}_{\text {calix }}\right), 7.27$ (d, J=5 Hz, 3H, $\left.\mathrm{ArH}_{\text {cap }}\right), 7.57$ (s, 3H, ArH $\left.\mathrm{cap}\right) .{ }^{13} \mathrm{C} \mathrm{NMR}\left(75 \mathrm{MHz}, \mathrm{CDCl}_{3}\right) \delta 29.6\left(\mathrm{CH}_{2}\right), 31.3\left(\mathrm{CH}_{3}\right), 31.8\left(\mathrm{CH}_{3}\right)$, $34.1(\mathrm{C}), 34.4(\mathrm{C}), 50.2\left(\mathrm{CH}_{2}\right), 52.2\left(\mathrm{CH}_{2}\right), 60.1\left(\mathrm{CH}_{3}\right), 68.5\left(\mathrm{CH}_{2}\right), 72.9\left(\mathrm{CH}_{2}\right), 112.2(\mathrm{CH}), 120.0$ $(\mathrm{CH}), 120.5(\mathrm{CH}), 123.5(\mathrm{CH}), 128.1(\mathrm{CH}), 128.2(\mathrm{CH}), 128.9(\mathrm{C}), 130.8(\mathrm{CH}), 133.2(\mathrm{C}), 133.6$ (C), 139.3 (C), 145.7(0) (C), 145.7(2) (C), 152.3 (C), 154.6 (C), 157.1 (C). Anal. Calcd for $\mathrm{C}_{105} \mathrm{H}_{129} \mathrm{~N}_{3} \mathrm{O}_{9} .2 \mathrm{H}_{2} \mathrm{O}: \mathrm{C}, 78.18 ; \mathrm{H}, 8.31 ; \mathrm{N}, 2.60$. Found: C, 78.12; H, 8.51; N, 2.53.

Calix[6]tris-imine 8: IR (KBr): $v 3700$ to 3100, 2961, 1641, 1599, $1482 \mathrm{~cm}^{-1} .{ }^{1} \mathrm{H}$ NMR (300 MHz, $\left.\mathrm{CDCl}_{3}\right) \delta 0.74(\mathrm{~s}, 27 \mathrm{H}, t \mathrm{Bu}), 1.37(\mathrm{~s}, 27 \mathrm{H}, t \mathrm{Bu}), 1.88(\mathrm{~s}, 9 \mathrm{H}, \mathrm{OMe}), 3.34\left(\mathrm{~d}, J=15 \mathrm{~Hz}, 6 \mathrm{H}, \operatorname{ArCH} H_{e q}\right)$, $4.11\left(\mathrm{~s}, 12 \mathrm{H}, \mathrm{OCH}_{2} \mathrm{CH}_{2} \mathrm{~N}\right), 4.17\left(\mathrm{~s}, 6 \mathrm{H}, \mathrm{ArCH}_{2 \mathrm{cap}}\right), 4.51\left(\mathrm{~d}, J=15 \mathrm{~Hz}, 6 \mathrm{H}, \mathrm{ArCH}_{a x}\right), 6.58(\mathrm{~s}, 6 \mathrm{H}$, $\mathrm{ArH}_{\text {calix }}$ ), 7.15 (s, 3H, $\left.\operatorname{ArH}_{\text {cap }}\right), 7.15-7.35$ (m, 9H, $\left.\operatorname{ArH}_{\text {cap }}\right), 7.21$ (s, 6H, $\left.\operatorname{ArH}_{\text {calix }}\right), 7.75$ (d, J = 8 Hz, $\left.3 \mathrm{H}, \mathrm{ArH}_{\mathrm{cap}}\right), 9.00(\mathrm{~s}, 3 \mathrm{H}, \mathrm{HC}=\mathrm{N}) .{ }^{13} \mathrm{C} \mathrm{NMR}\left(75 \mathrm{MHz}, \mathrm{CDCl}_{3}\right) \delta 29.7\left(\mathrm{CH}_{2}\right), 31.3\left(\mathrm{CH}_{3}\right), 31.8\left(\mathrm{CH}_{3}\right)$, $34.1(\mathrm{C}), 34.4(\mathrm{C}), 38.8\left(\mathrm{CH}_{2}\right), 59.5\left(\mathrm{CH}_{3}\right), 61.7\left(\mathrm{CH}_{2}\right), 71.1\left(\mathrm{CH}_{2}\right), 123.4(\mathrm{CH}), 126.7(\mathrm{CH}), 127.6$ $(\mathrm{CH}), 127.9(\mathrm{CH}), 128.0(\mathrm{CH}), 130.3(\mathrm{CH}), 130.6(\mathrm{CH}), 133.2(\mathrm{C}), 133.7(\mathrm{C}), 135.1(\mathrm{C}), 141.4(\mathrm{C})$, 141.7 (C), 145.5 (C), 145.8 (C), 151.5 (C), 154.9 (C), 163.6 (C).

Calix[6]tamb 9: $\mathrm{mp} 275^{\circ} \mathrm{C}$ (dec). IR (KBr): $v 3720$ to 3100, 2961, 1482, $1385 \mathrm{~cm}^{-1} .{ }^{1} \mathrm{H}$ NMR (300 $\left.\mathrm{MHz}, \mathrm{CDCl}_{3}\right) \delta 0.77(\mathrm{~s}, 27 \mathrm{H}, t \mathrm{Bu}), 1.37(\mathrm{~s}, 27 \mathrm{H}, t \mathrm{Bu}), 2.10(\mathrm{~s}, 9 \mathrm{H}, \mathrm{OMe}), 3.09\left(\mathrm{~s}, 6 \mathrm{H}, \mathrm{OCH}_{2} \mathrm{CH}_{2} \mathrm{~N}\right)$, $3.38\left(\mathrm{~d}, J=15 \mathrm{~Hz}, 6 \mathrm{H}, \mathrm{ArCH}_{e q}\right), 4.03\left(\mathrm{~s}, 6 \mathrm{H}, \mathrm{OCH}_{2} \mathrm{CH}_{2} \mathrm{~N}\right), 4.05\left(\mathrm{~s}, 6 \mathrm{H}, \mathrm{ArCH}_{2} \mathrm{~N}\right), 4.15(\mathrm{~s}, 6 \mathrm{H}$, $\left.\mathrm{ArCH}_{2 \text { cap }}\right), 4.58$ (d, $\left.J=15 \mathrm{~Hz}, 6 \mathrm{H}, \mathrm{ArCH}_{a x}\right), 6.63$ (s, 6H, $\left.\mathrm{ArH}_{\text {calix }}\right), 7.03$ (s, 3H, ArH $\left.\mathrm{Aap}_{\text {) }}\right), 7.08$ (m, 3H,

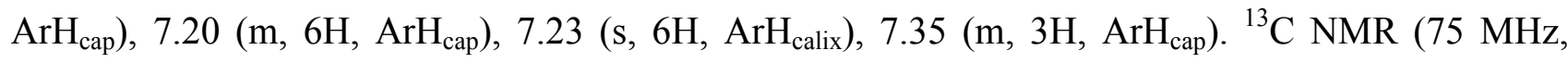
$\left.\mathrm{CDCl}_{3}\right) \delta 29.7\left(\mathrm{CH}_{2}\right), 31.3\left(\mathrm{CH}_{3}\right), 31.8\left(\mathrm{CH}_{3}\right), 34.2(\mathrm{C}), 34.4(\mathrm{C}), 38.5\left(\mathrm{CH}_{2}\right), 49.8\left(\mathrm{CH}_{2}\right), 52.6$ $\left(\mathrm{CH}_{2}\right), 60.0\left(\mathrm{CH}_{3}\right), 72.7\left(\mathrm{CH}_{2}\right), 123.7(\mathrm{CH}), 126.5(\mathrm{CH}), 127.5(\mathrm{CH}), 128.0(8)(\mathrm{CH}), 128.1(5)(\mathrm{CH})$, $130.2(\mathrm{CH}), 130.5(\mathrm{CH}), 133.2(\mathrm{C}), 133.8(\mathrm{C}), 138.2(\mathrm{C}), 140.2(\mathrm{C}), 141.6(\mathrm{C}), 145.8(8)(\mathrm{C})$, 145.9(4) (C), 151.5 (C), 154.6 (C). Anal. Calcd for $\mathrm{C}_{105} \mathrm{H}_{129} \mathrm{~N}_{3} \mathrm{O}_{6} \cdot \mathrm{H}_{2} \mathrm{O}: \mathrm{C}, 81.51 ; \mathrm{H}, 8.53$; N, 2.72. Found: C, 81.68; H, 8.67; N, 2.64.

Complex 9.3H ${ }^{+}$IMI: ${ }^{1} \mathrm{H}$ NMR (300 MHz, $\left.\mathrm{CDCl}_{3}\right) \delta 0.24\left(\mathrm{~s}, 4 \mathrm{H}, \mathrm{CH}_{2 \mathrm{IMIin}}\right), 0.76(\mathrm{~s}, 27 \mathrm{H}, t \mathrm{Bu}), 1.41$ $(\mathrm{s}, 27 \mathrm{H}, t \mathrm{Bu}), 3.18\left(\mathrm{~s}, 6 \mathrm{H}, \mathrm{OCH}_{2} \mathrm{CH}_{2} \mathrm{~N}\right), 3.46\left(\mathrm{~s}, 6 \mathrm{H}, \mathrm{ArCH}_{2} \mathrm{~N}\right), 3.59\left(\mathrm{~d}, J=15 \mathrm{~Hz}, 6 \mathrm{H}, \operatorname{ArCH}_{e q}\right)$, $3.74(\mathrm{~s}, 9 \mathrm{H}, \mathrm{OMe}), 4.07$ (s, 6H, $\left.\mathrm{ArCH}_{2 \text { cap }}\right), 4.15\left(\mathrm{~s}, 6 \mathrm{H}, \mathrm{OCH}_{2} \mathrm{CH}_{2} \mathrm{~N}\right), 4.42(\mathrm{~d}, J=15 \mathrm{~Hz}, 6 \mathrm{H}$, $\left.\operatorname{ArCH}_{a x}\right), 4.62\left(\mathrm{~s}, 2 \mathrm{H}, \mathrm{NH}_{\mathrm{IMIin}}\right), 6.59\left(\mathrm{~s}, 6 \mathrm{H}, \mathrm{ArH}_{\text {calix }}\right), 6.69$ (s, 3H, ArH $\left.\mathrm{Aap}\right), 7.36\left(\mathrm{~s}, 15 \mathrm{H}, \mathrm{ArH}_{\text {cap }}+\right.$ $\left.\mathrm{ArH}_{\text {calix }}\right), 7.75\left(\mathrm{~s}_{\mathrm{b}}, 3 \mathrm{H}, \mathrm{ArH}_{\mathrm{cap}}\right)$. 


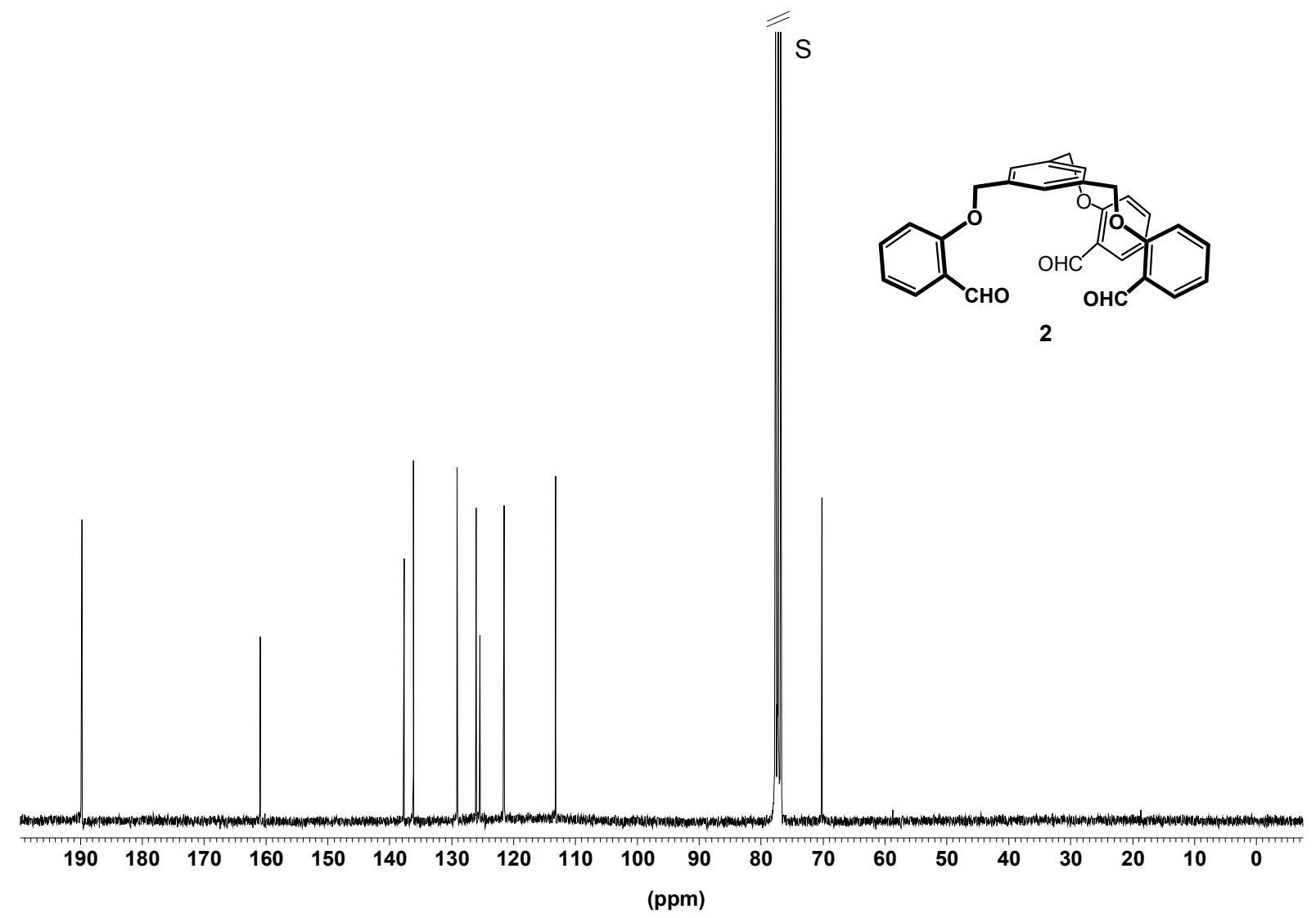

Figure S1. ${ }^{13} \mathrm{C}$ NMR spectrum $\left(75 \mathrm{MHz}, \mathrm{CDCl}_{3}\right)$ of compound 2. Solvent has been labeled "S".

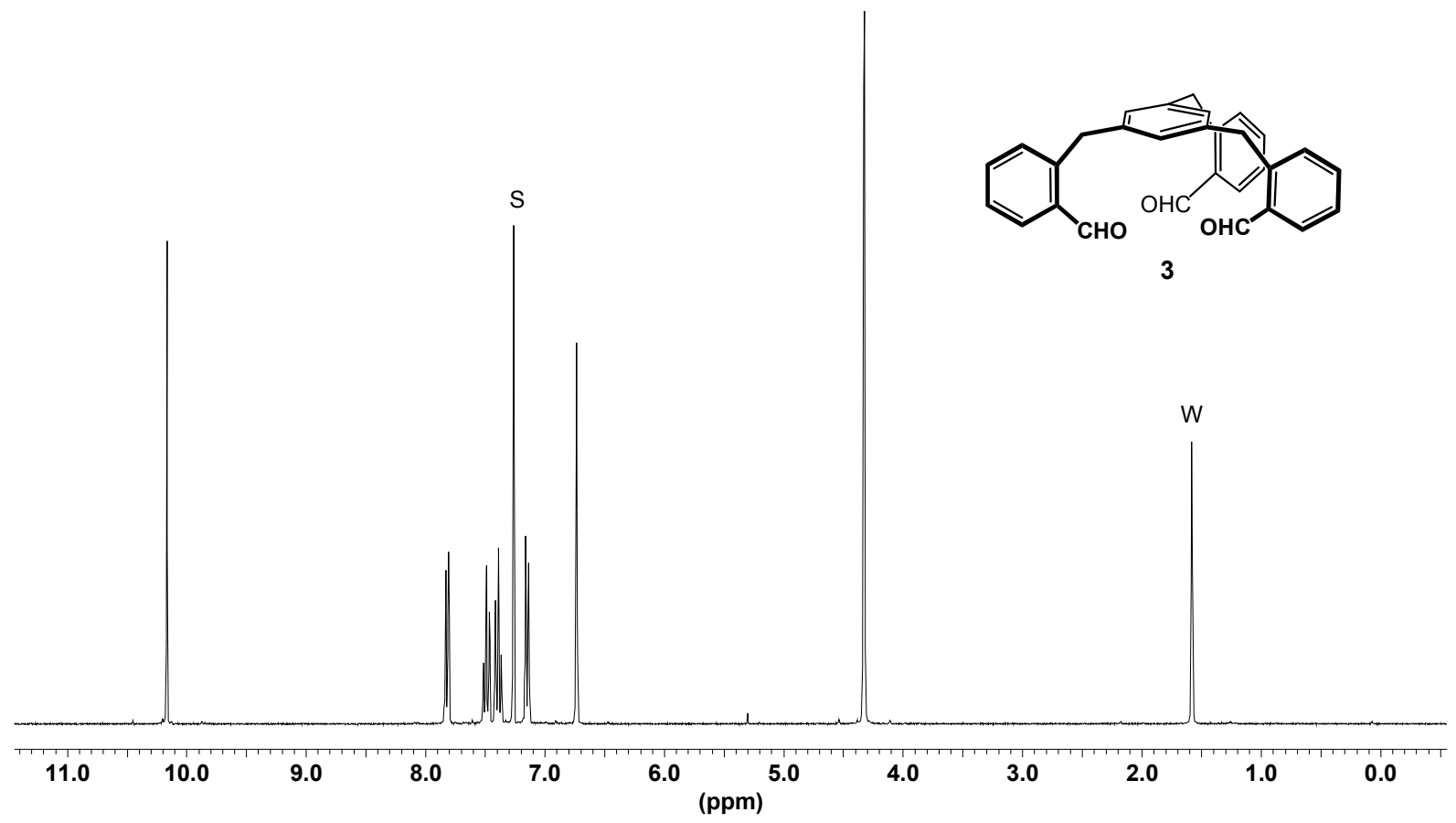

Figure S2. ${ }^{1} \mathrm{H}$ NMR spectrum $\left(300 \mathrm{MHz}, \mathrm{CDCl}_{3}\right)$ of compound 3. Solvent and water have been labeled "S" and "W", respectively. 


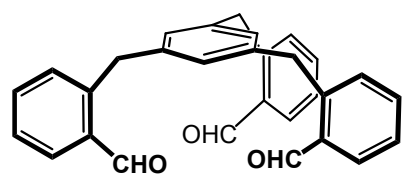

3

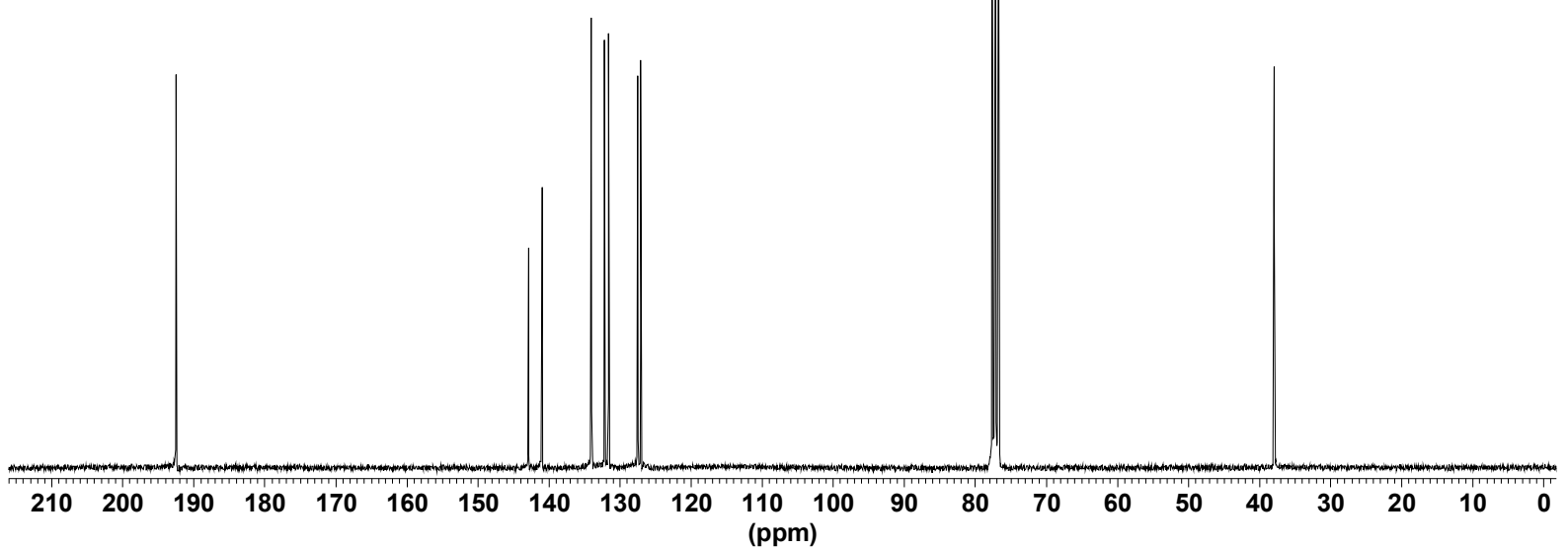

Figure S3. ${ }^{13} \mathrm{C}$ NMR spectrum $\left(75 \mathrm{MHz}, \mathrm{CDCl}_{3}\right)$ of compound 3. Solvent has been labeled "S".

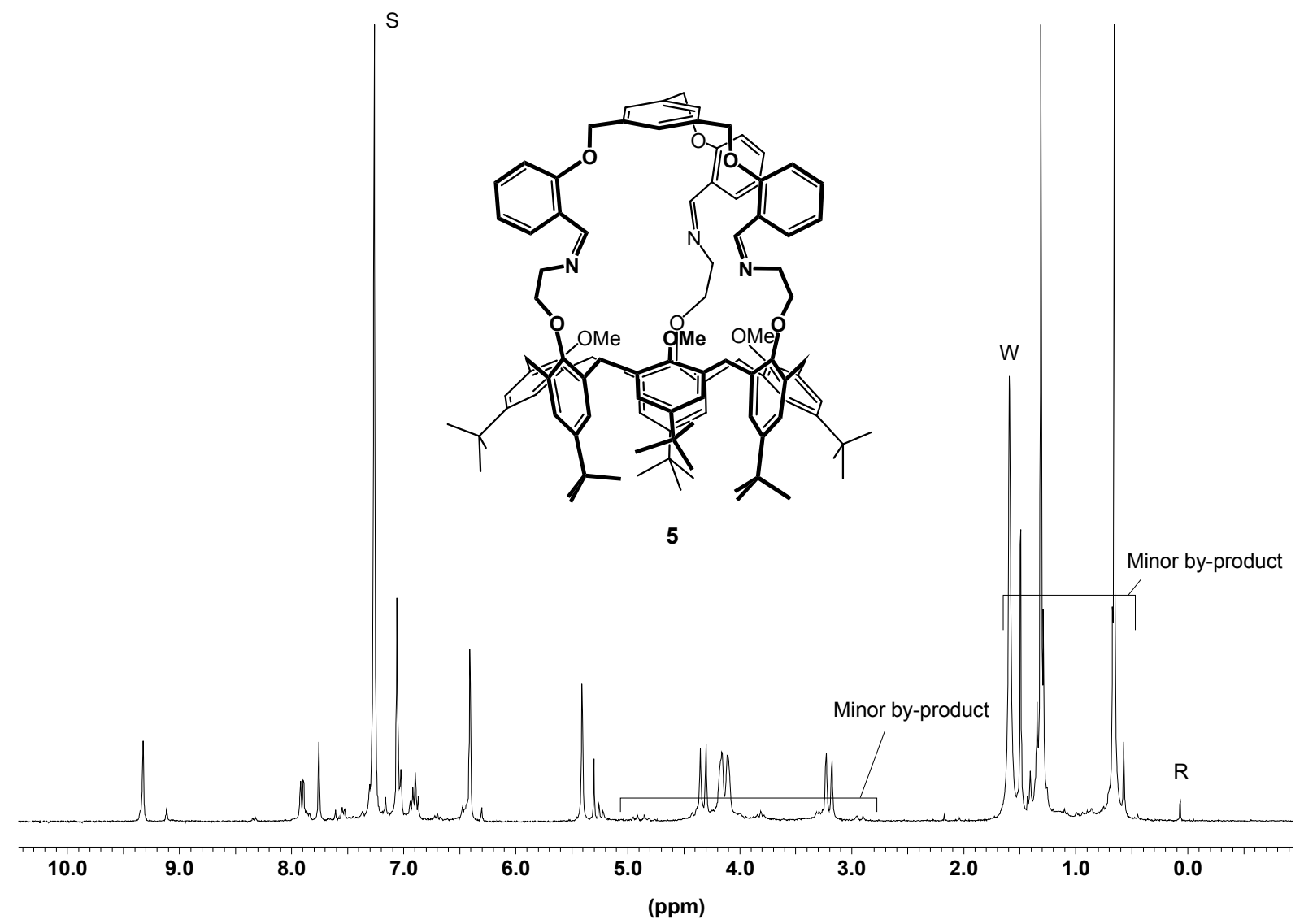

Figure S4. ${ }^{1} \mathrm{H}$ NMR spectrum $\left(300 \mathrm{MHz}, \mathrm{CDCl}_{3}\right)$ of crude compound 5. Solvent, reference and water have been labeled "S", "R" and "W", respectively. 

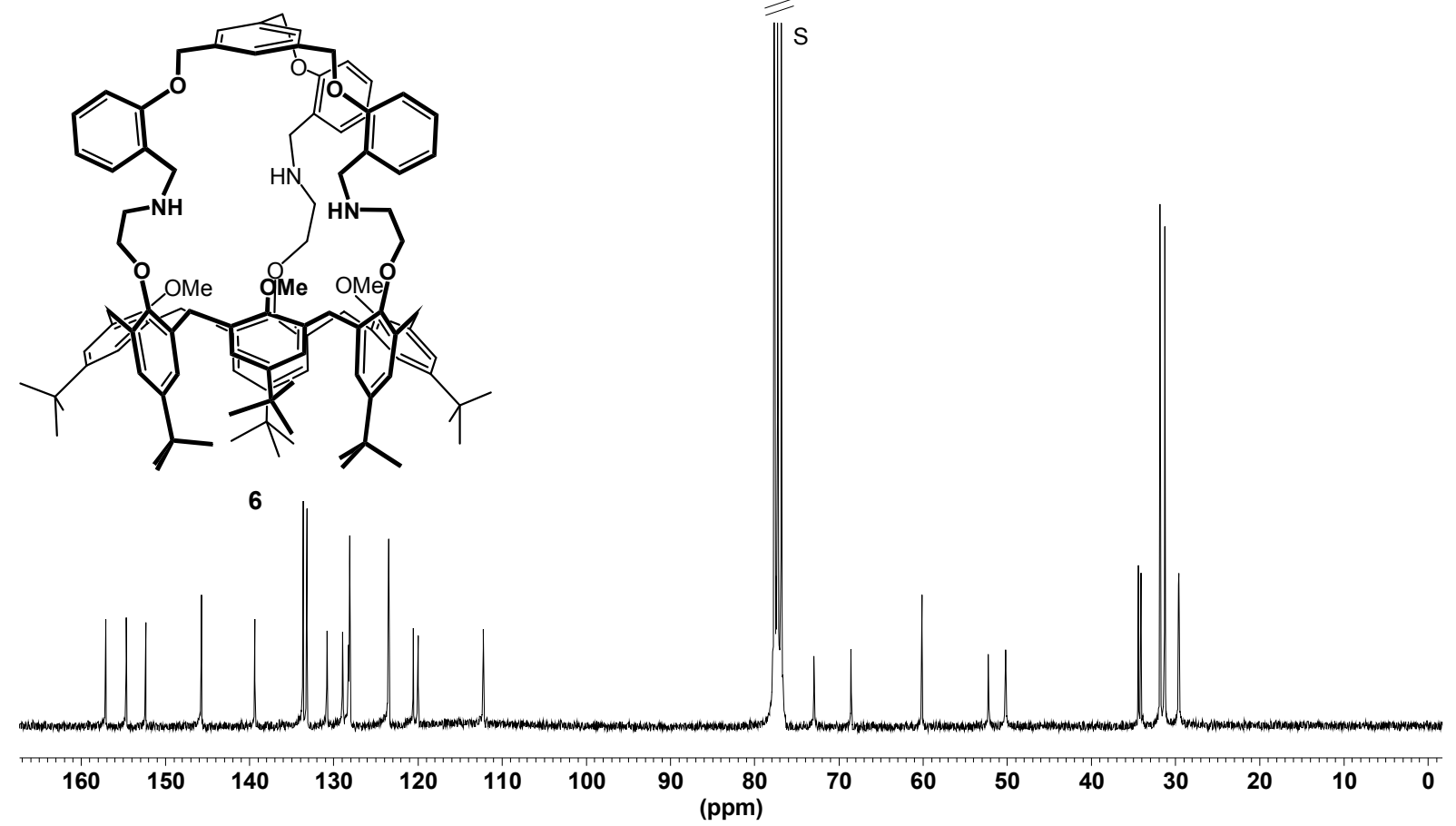

Figure S5. ${ }^{13} \mathrm{C}$ NMR spectrum $\left(75 \mathrm{MHz}, \mathrm{CDCl}_{3}\right)$ of calix[6]tampo 6. Solvent has been labeled "S".
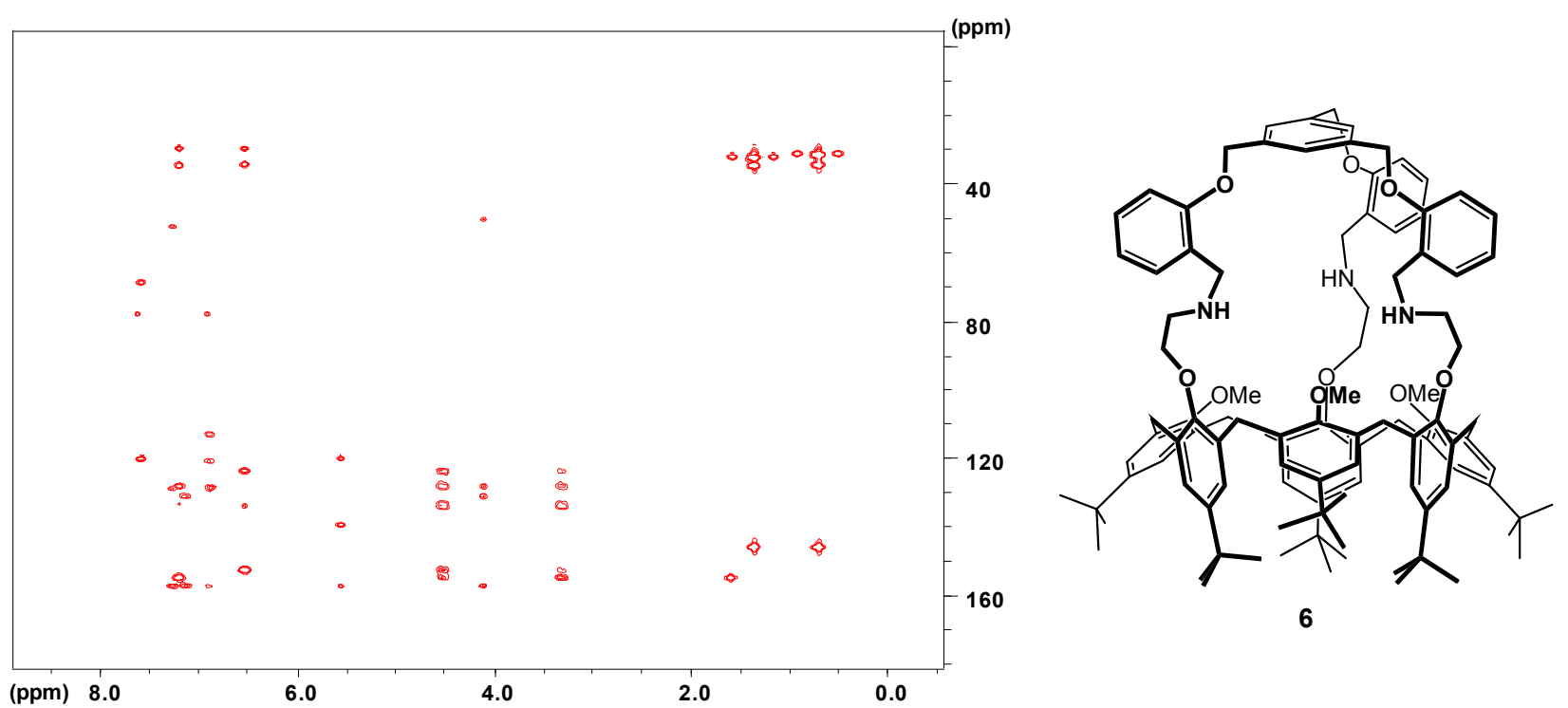

Figure S6. HMBC spectrum $\left(\mathrm{CDCl}_{3}\right)$ of calix[6]tampo 6 . 


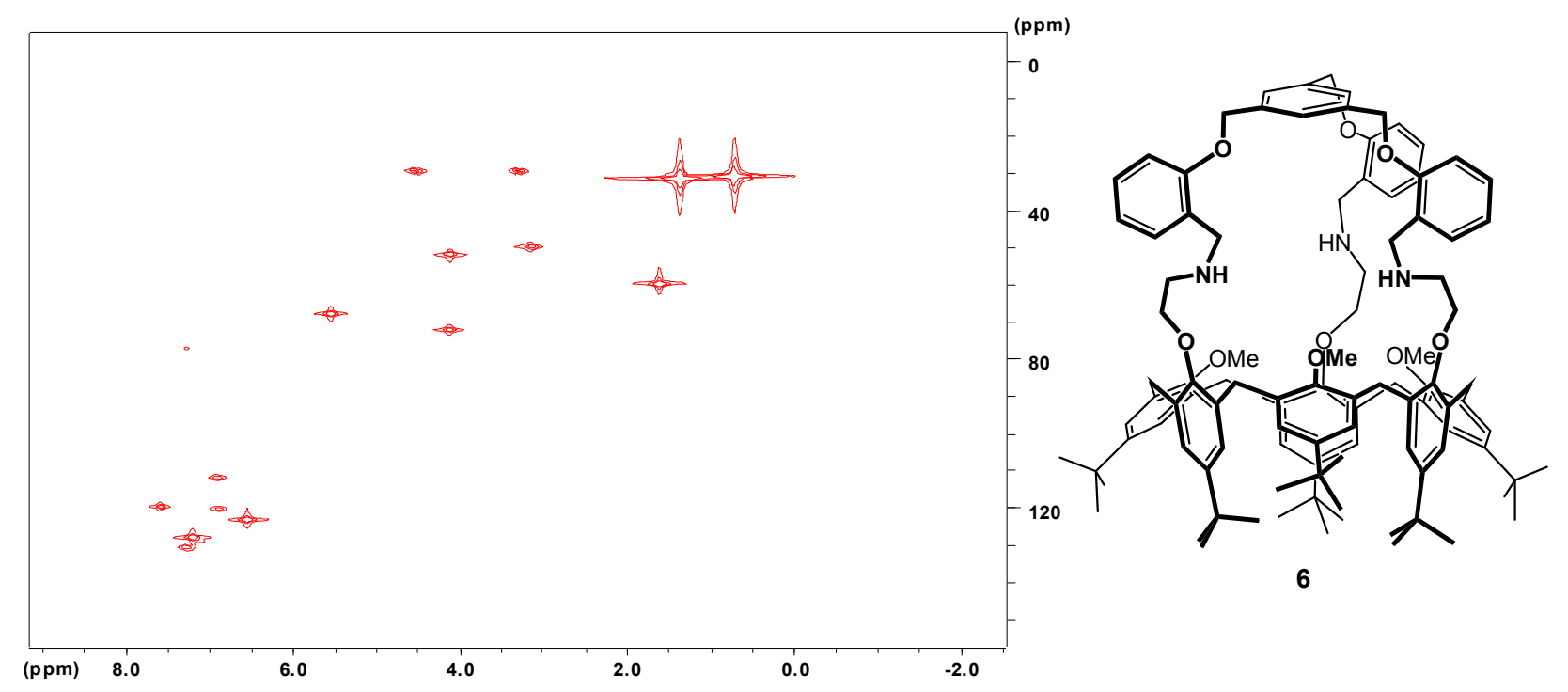

Figure S7. HMQC spectrum $\left(\mathrm{CDCl}_{3}\right)$ of calix[6]tampo 6.

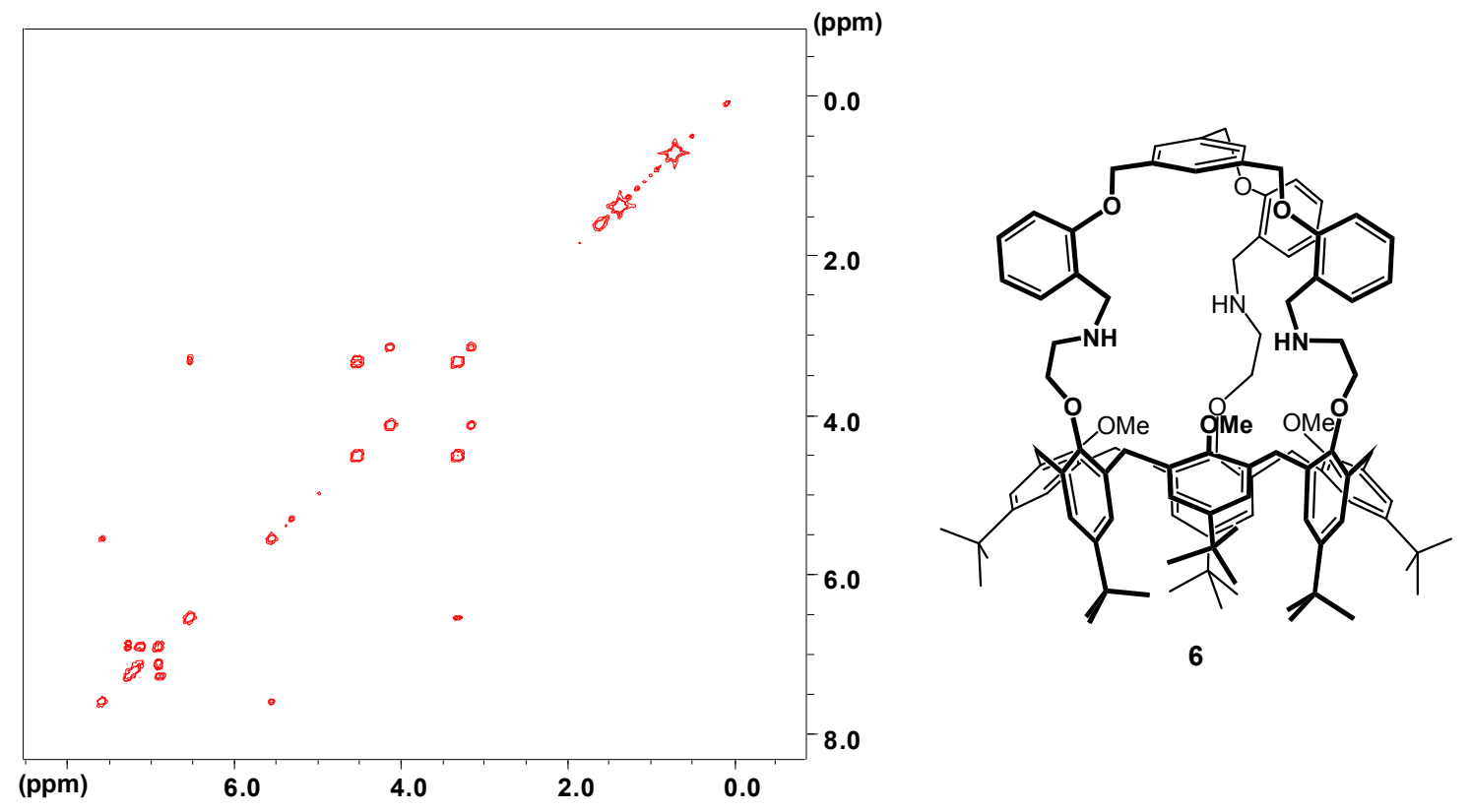

Figure S8. COSY spectrum $\left(\mathrm{CDCl}_{3}\right)$ of calix[6]tampo 6. 


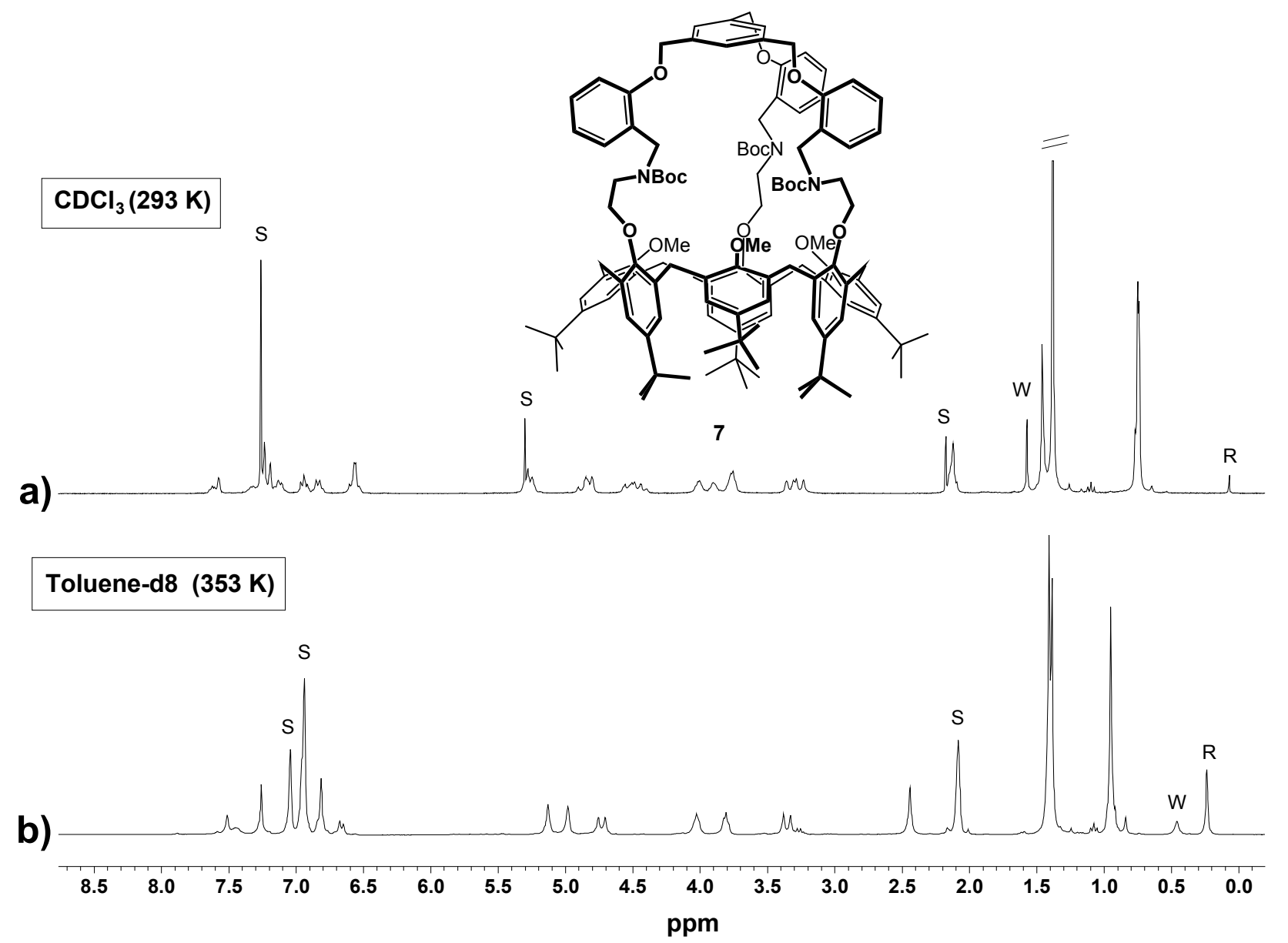

Figure S9. ${ }^{1} \mathrm{H}$ NMR spectra (300 MHz) of protected calix[6]tampo 7. a) in $\mathrm{CDCl}_{3}$ at $293 \mathrm{~K}$; b) in toluene-d8 at $353 \mathrm{~K}$. Solvents (spectrum a from the left: $\mathrm{CHCl}_{3}, \mathrm{CH}_{2} \mathrm{Cl}_{2}, \mathrm{CH}_{3} \mathrm{CN}$; spectrum b: toluene), reference and water have been labeled "S", "R" and "W", respectively.

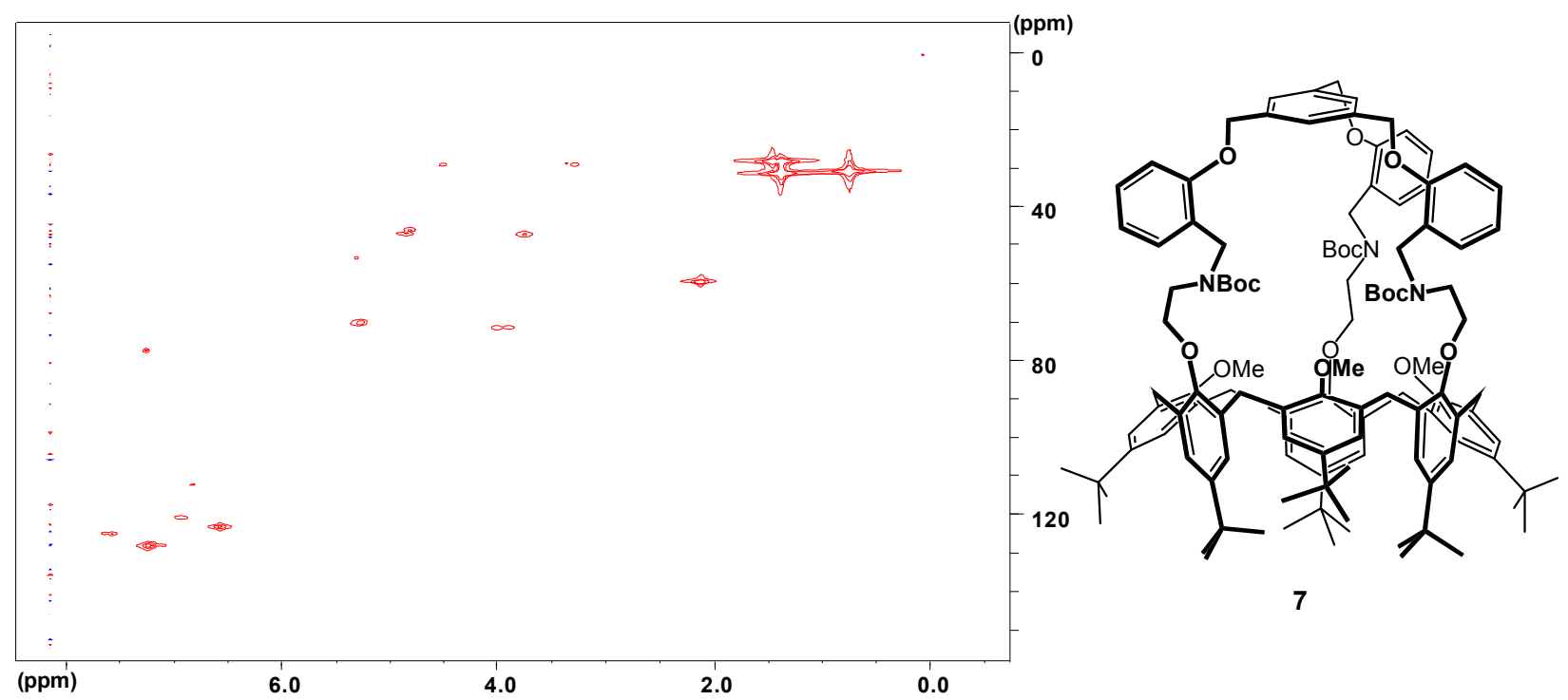

Figure S10. HMQC spectrum $\left(\mathrm{CDCl}_{3}\right)$ of protected calix[6]tampo 7. 

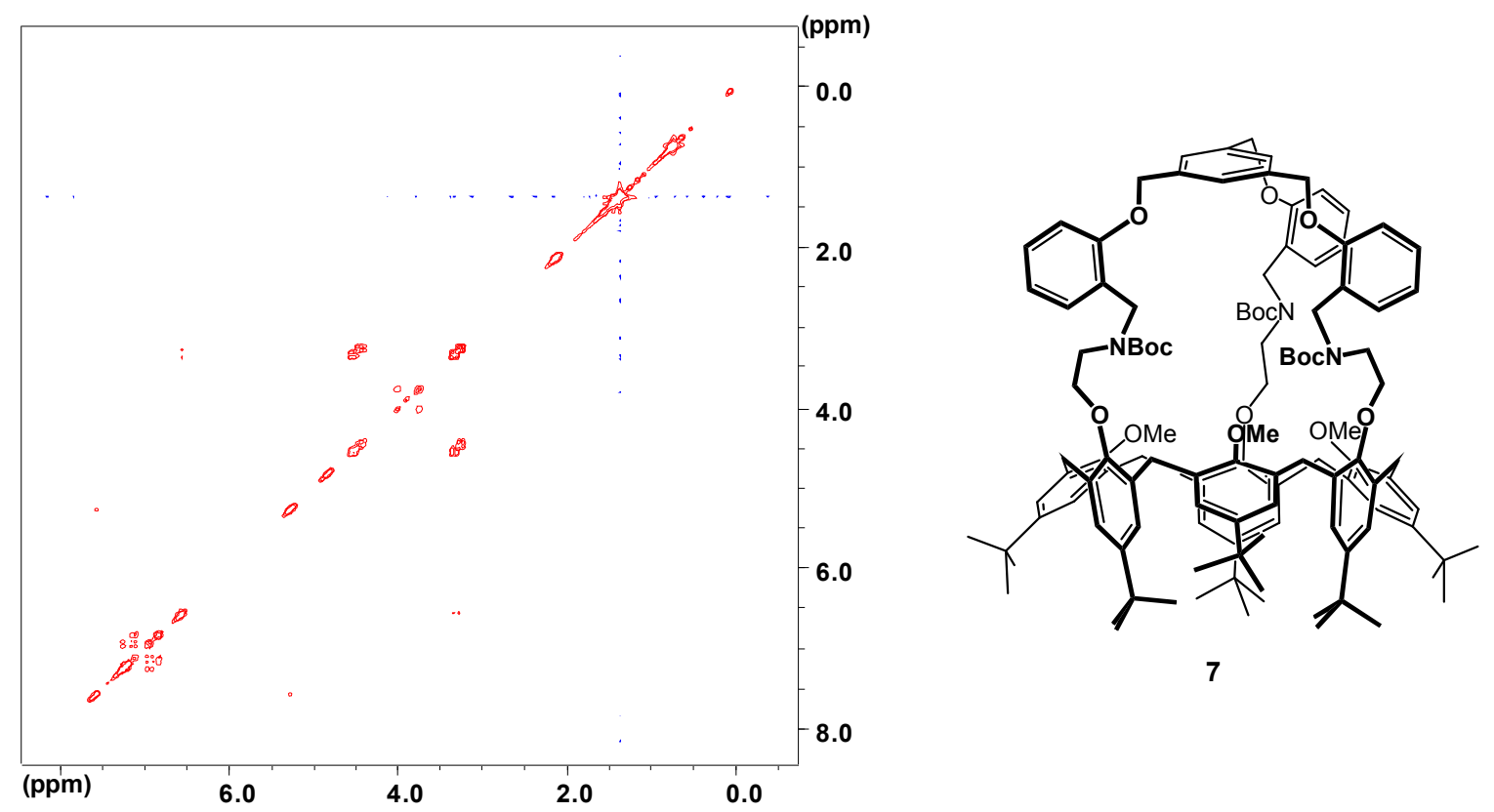

Figure S11. COSY spectrum $\left(\mathrm{CDCl}_{3}\right)$ of protected calix[6]tampo 7 .

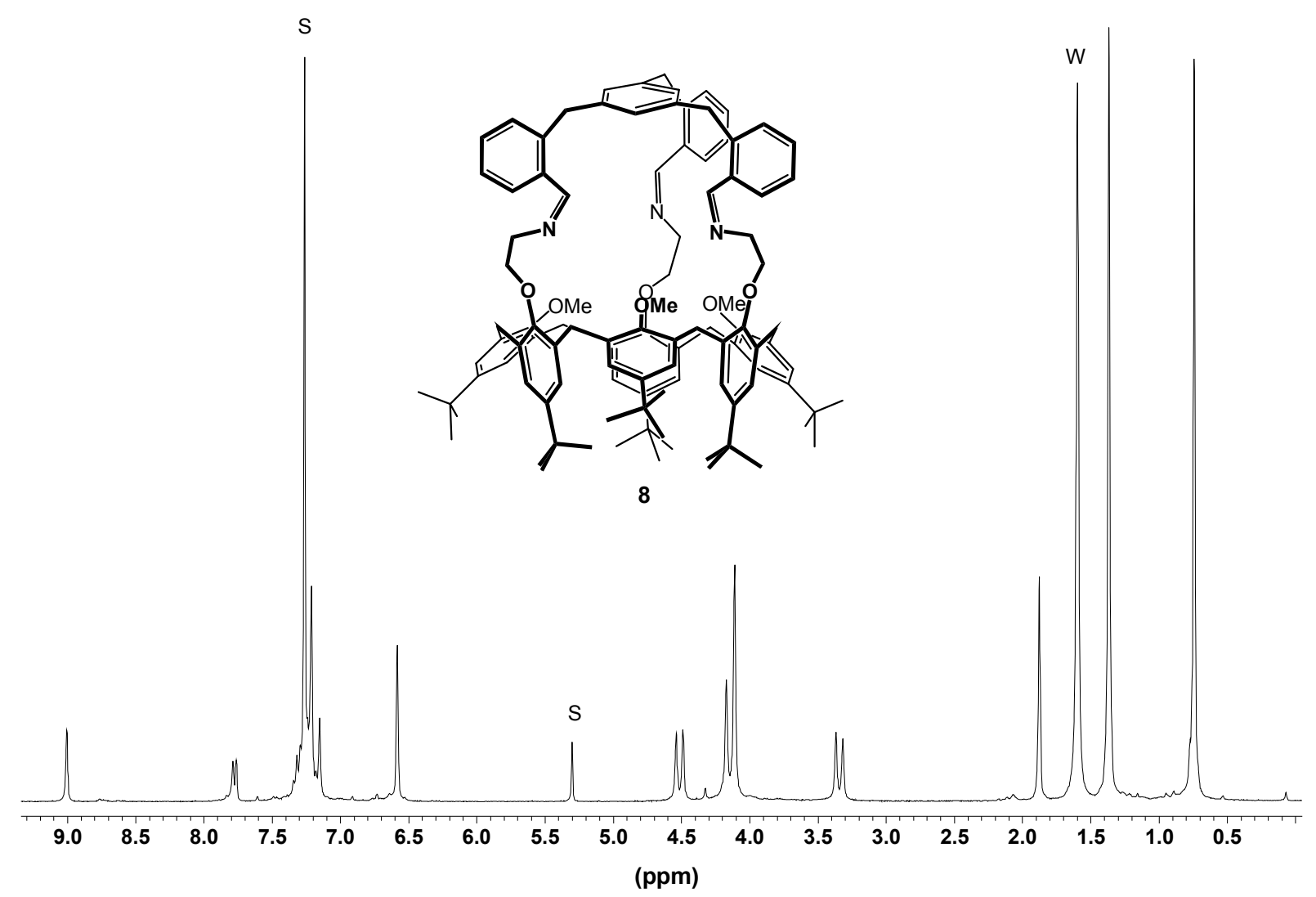

Figure S12. ${ }^{1} \mathrm{H}$ NMR spectrum $\left(300 \mathrm{MHz}, \mathrm{CDCl}_{3}\right.$ ) of crude compound 8. Solvents (from the left: $\mathrm{CHCl}_{3}, \mathrm{CH}_{2} \mathrm{Cl}_{2}$ ) and water have been labeled "S" and "W", respectively. 


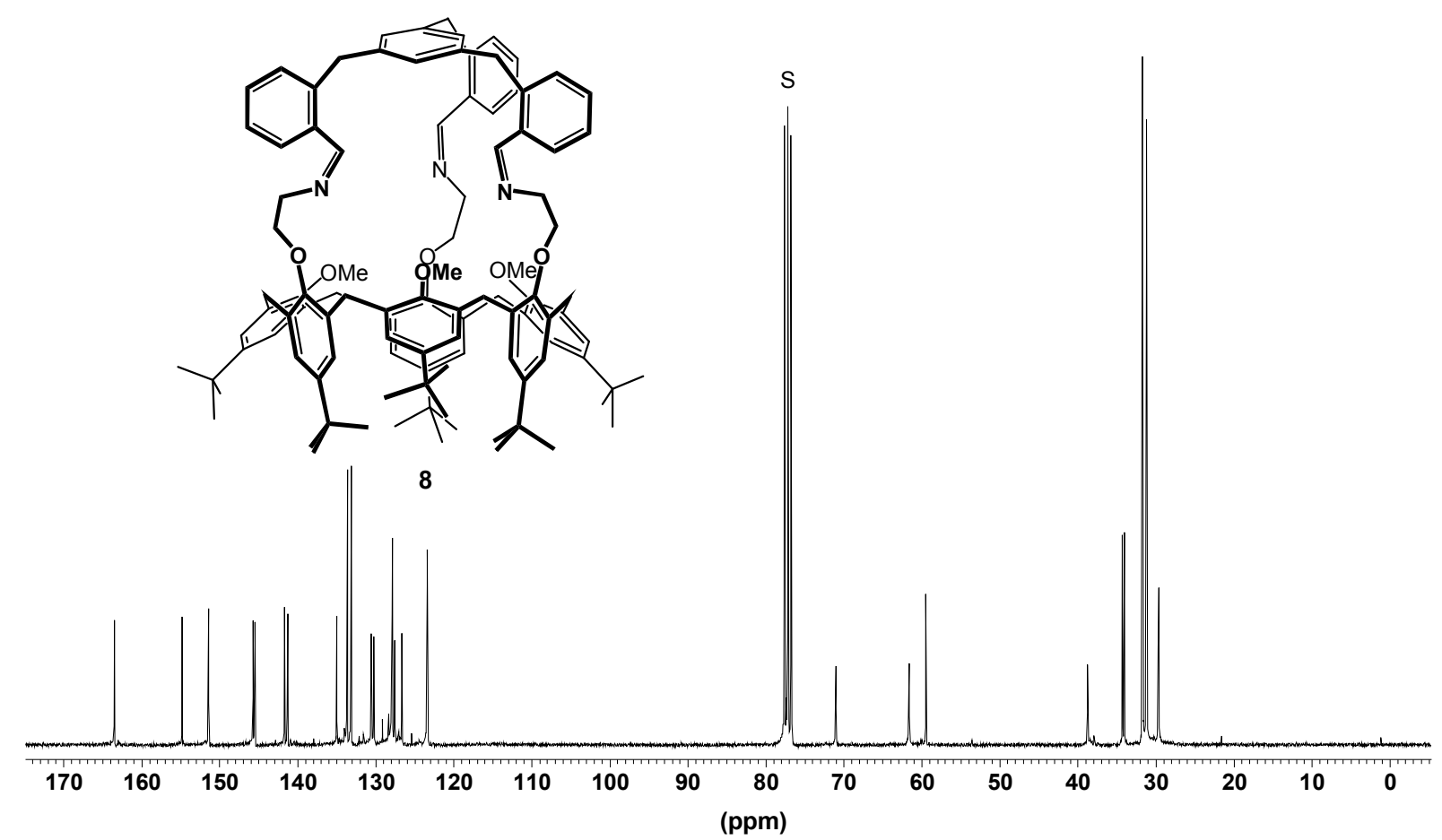

Figure S13. ${ }^{13} \mathrm{C}$ NMR spectrum $\left(75 \mathrm{MHz}, \mathrm{CDCl}_{3}\right.$ ) of crude compound 8 . Solvent has been labeled "S".

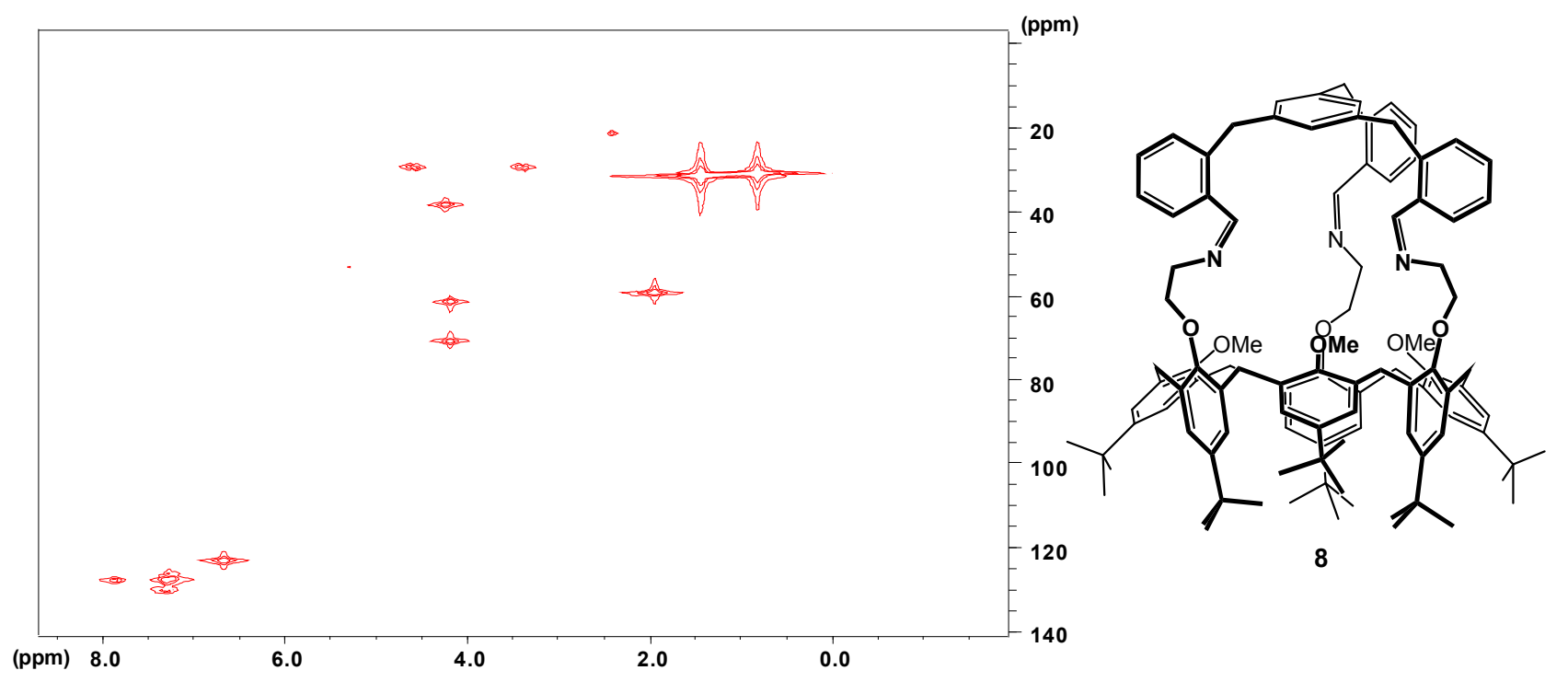

Figure S14. HMQC spectrum $\left(\mathrm{CDCl}_{3}\right)$ of crude compound 8 . 


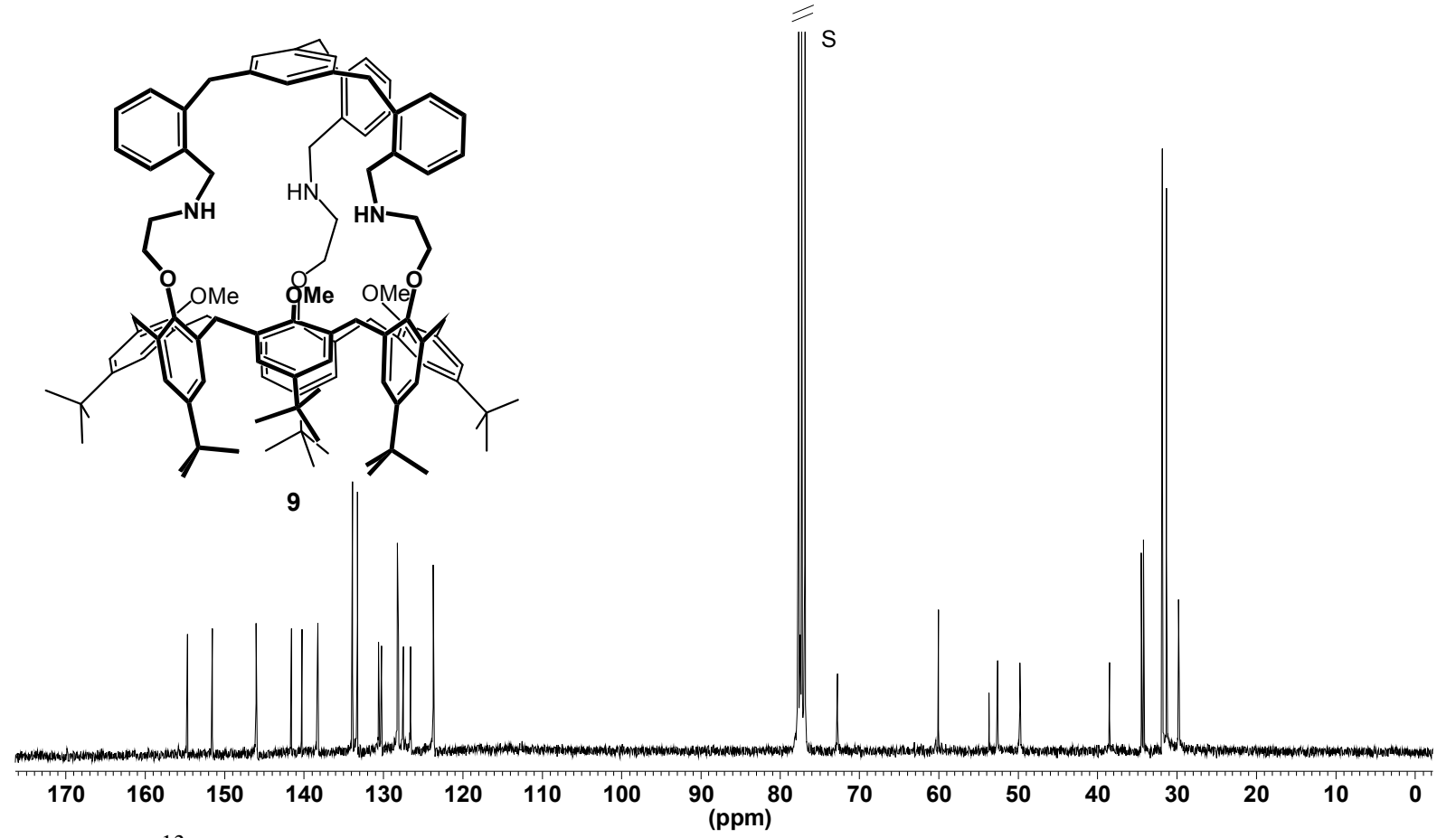

Figure S15. ${ }^{13} \mathrm{C}$ NMR spectrum $\left(75 \mathrm{MHz}, \mathrm{CDCl}_{3}\right)$ of calix[6]tamb 9. Solvent has been labeled "S".

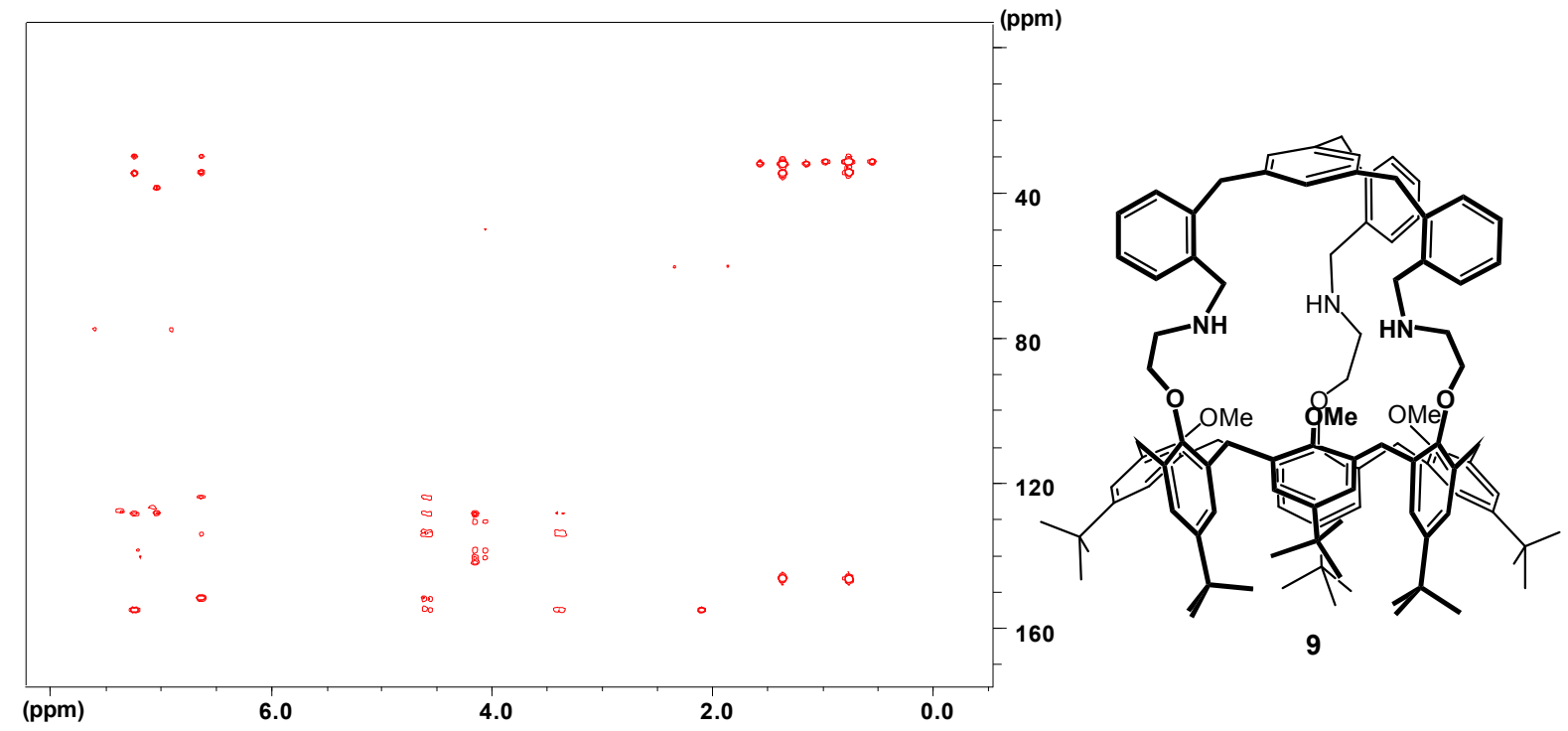

Figure S16. HMBC spectrum $\left(\mathrm{CDCl}_{3}\right)$ of calix[6]tamb 9. 


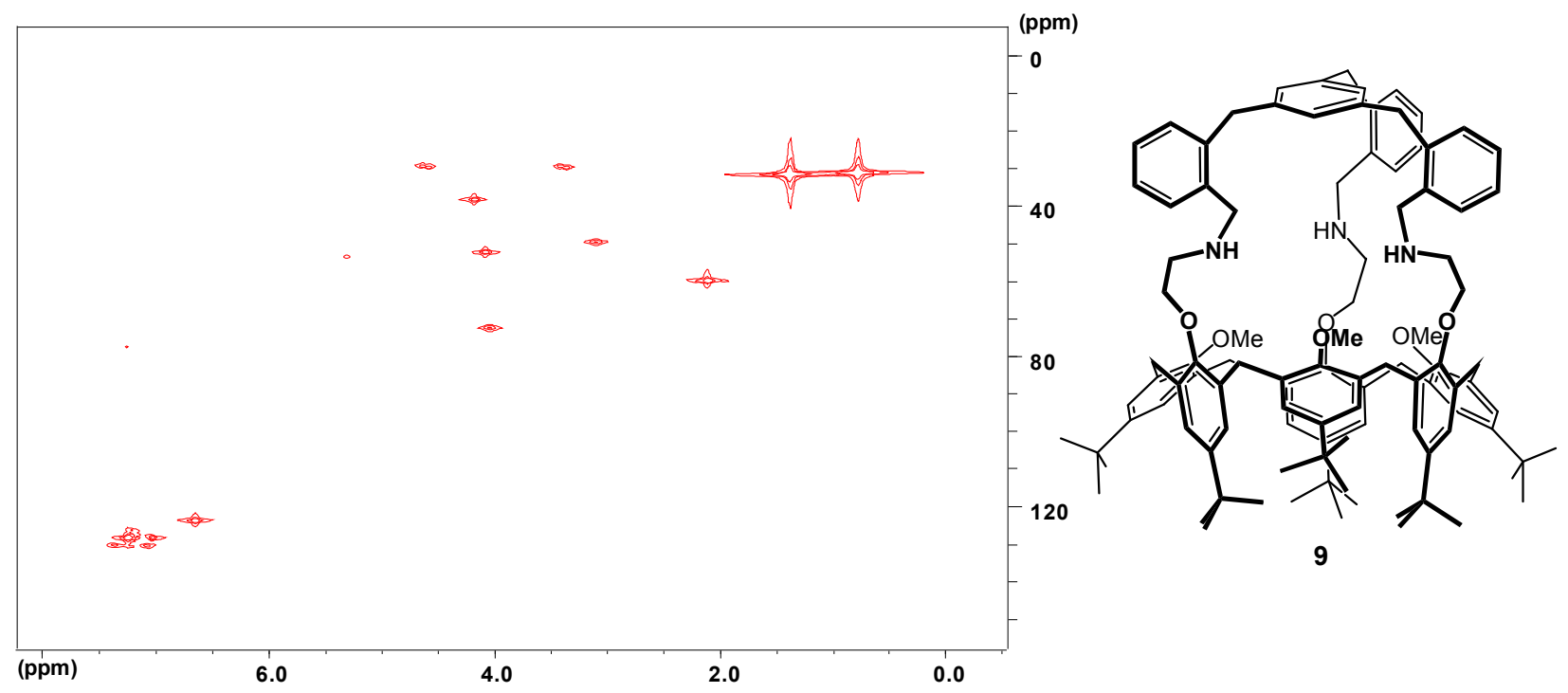

Figure S17. HMQC spectrum $\left(\mathrm{CDCl}_{3}\right)$ of calix[6]tamb 9.

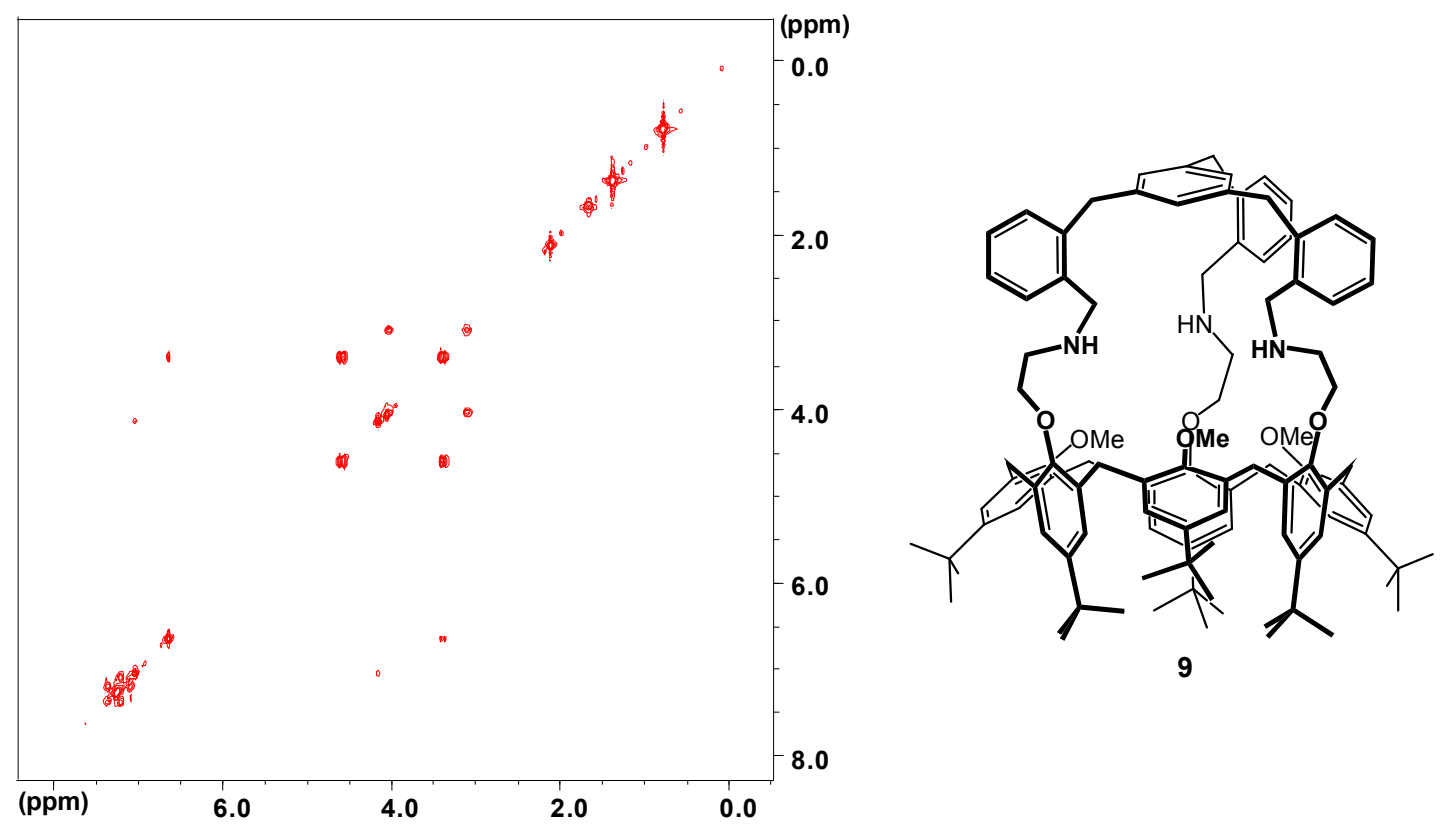

Figure S18. COSY spectrum $\left(\mathrm{CDCl}_{3}\right)$ of calix[6]tamb 9. 


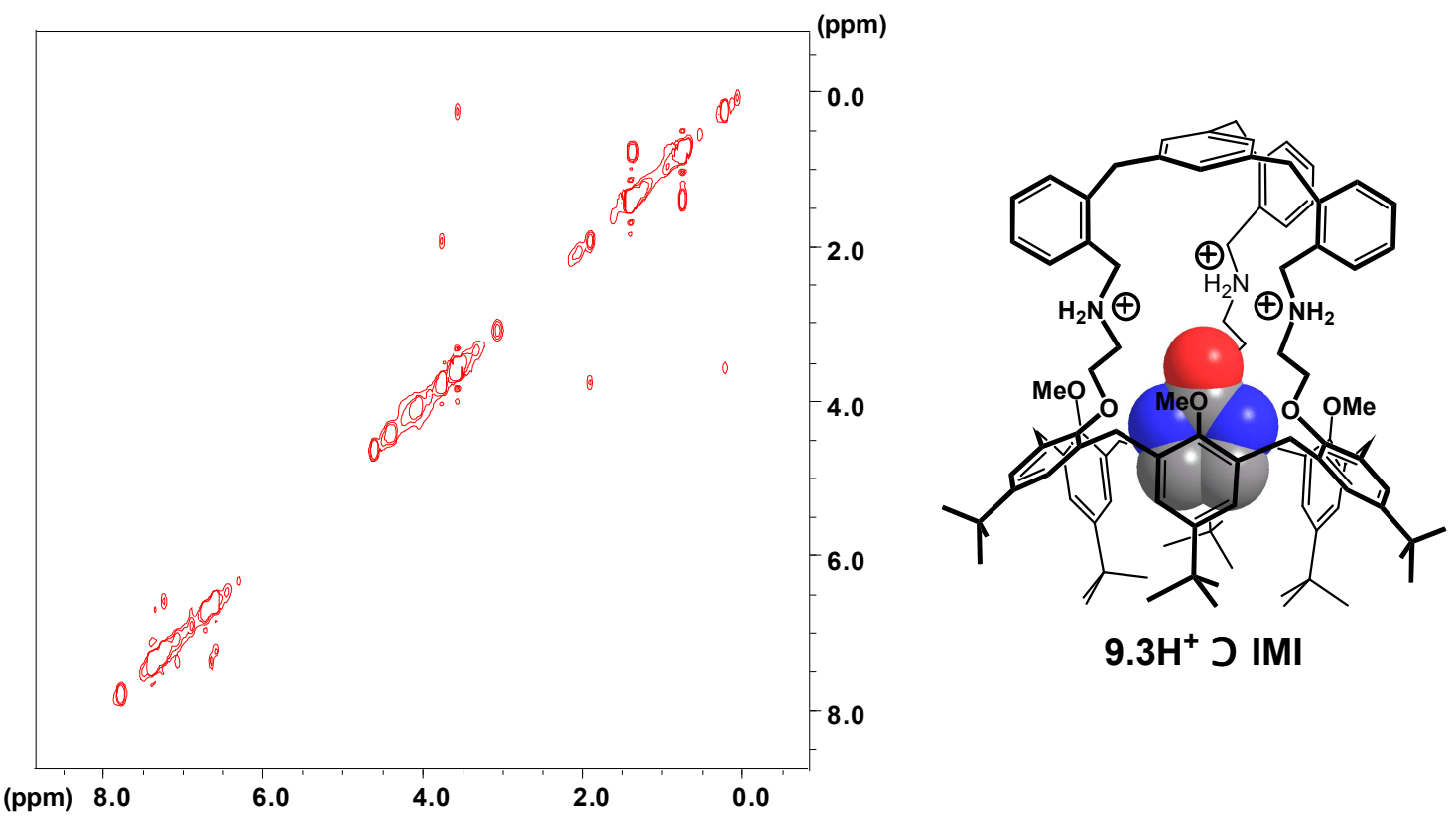

Figure S19. NOESY spectrum $\left(\mathrm{CDCl}_{3}\right)$ of the host-guest complex $9.3 \mathbf{H}^{+} \supset \mathbf{I M I}$.

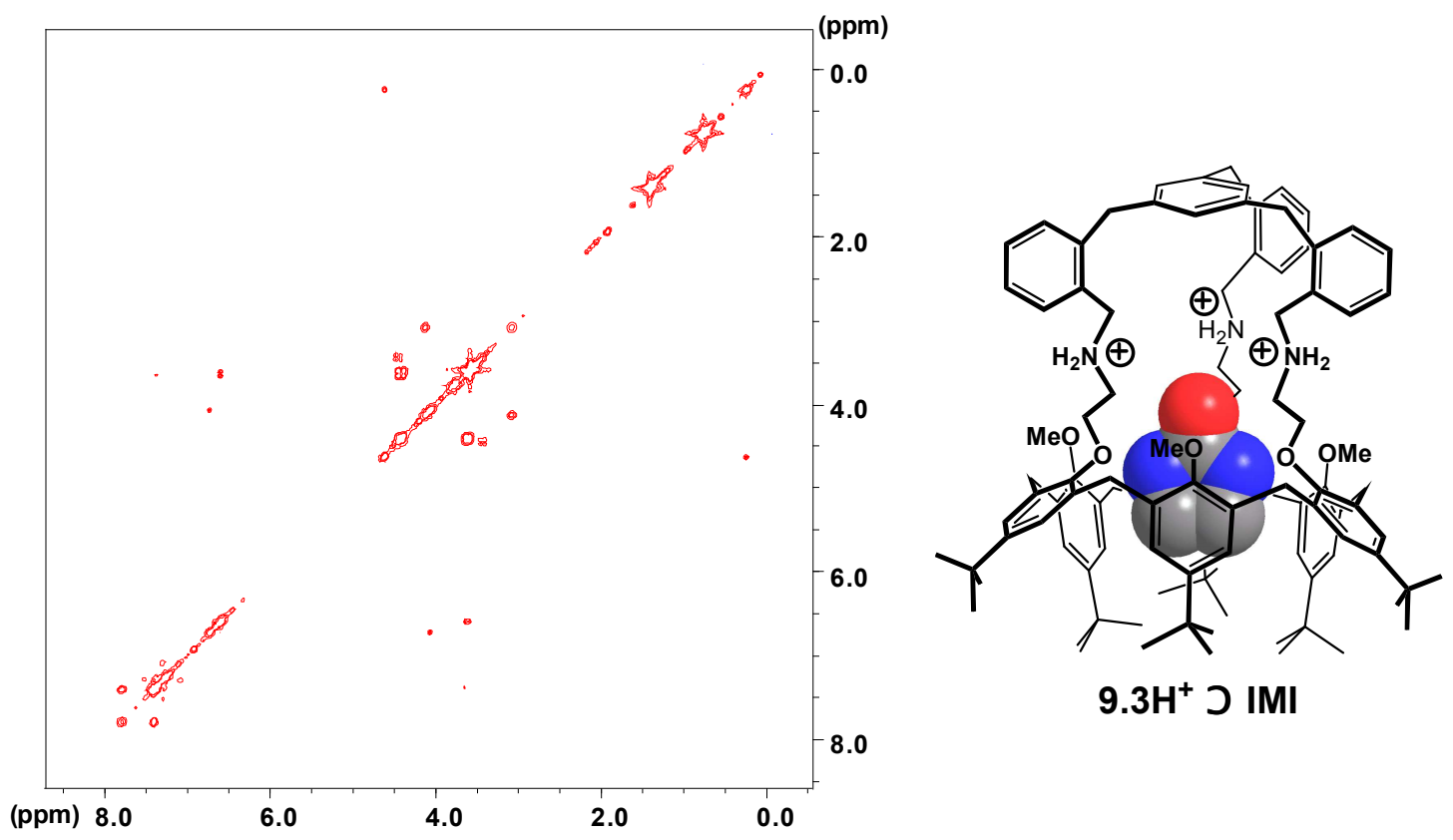

Figure S20. COSY spectrum $\left(\mathrm{CDCl}_{3}\right)$ of the host-guest complex $9.3 \mathbf{H}^{+} \supset \mathbf{I M I}$. 


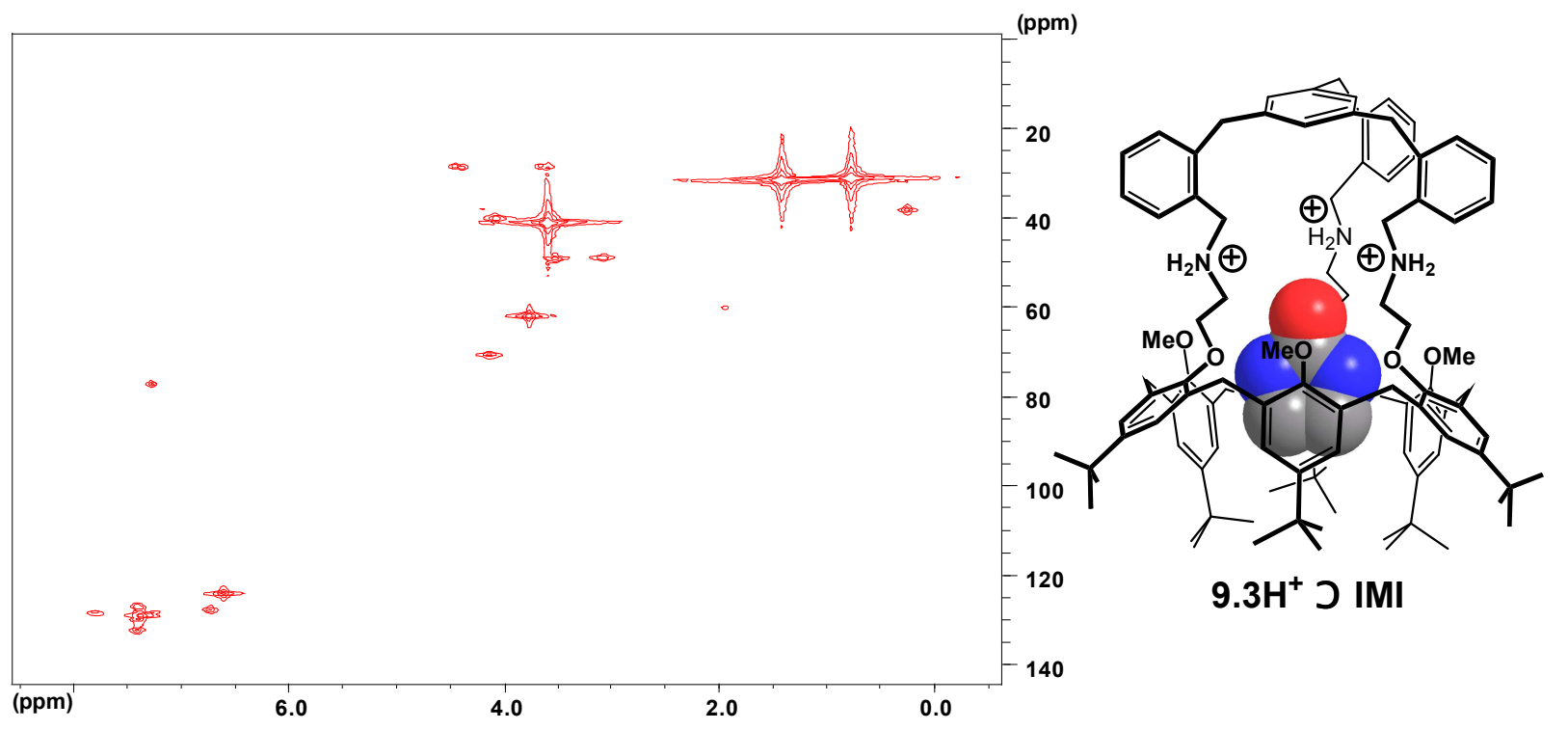

Figure S21. HMQC spectrum $\left(\mathrm{CDCl}_{3}\right)$ of the host-guest complex $9.3 \mathbf{H}^{+} \supset \mathbf{I M I}$.

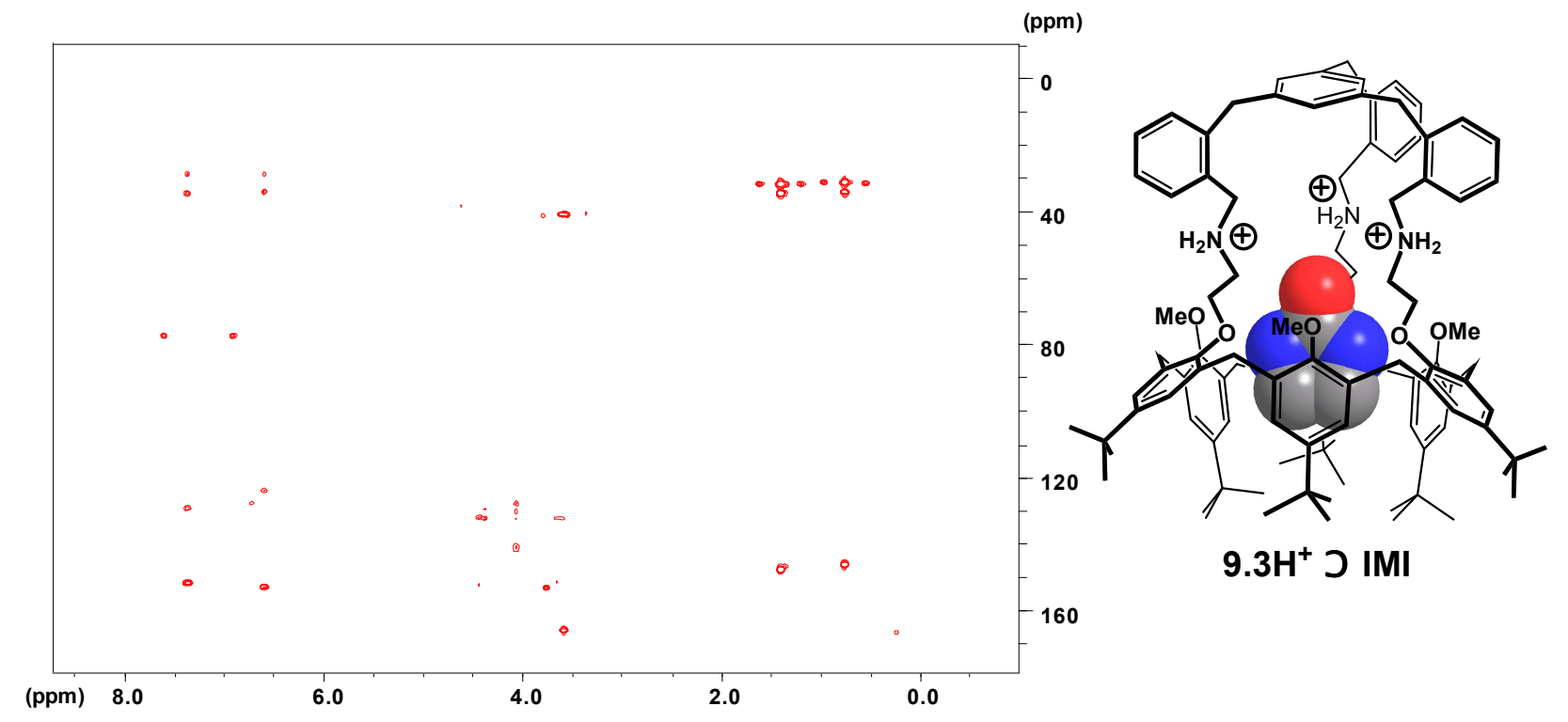

Figure S22. HMBC spectrum $\left(\mathrm{CDCl}_{3}\right)$ of the host-guest complex $\mathbf{9 . 3 \mathbf { H } ^ { + }} \supset \mathbf{I M I}$. 


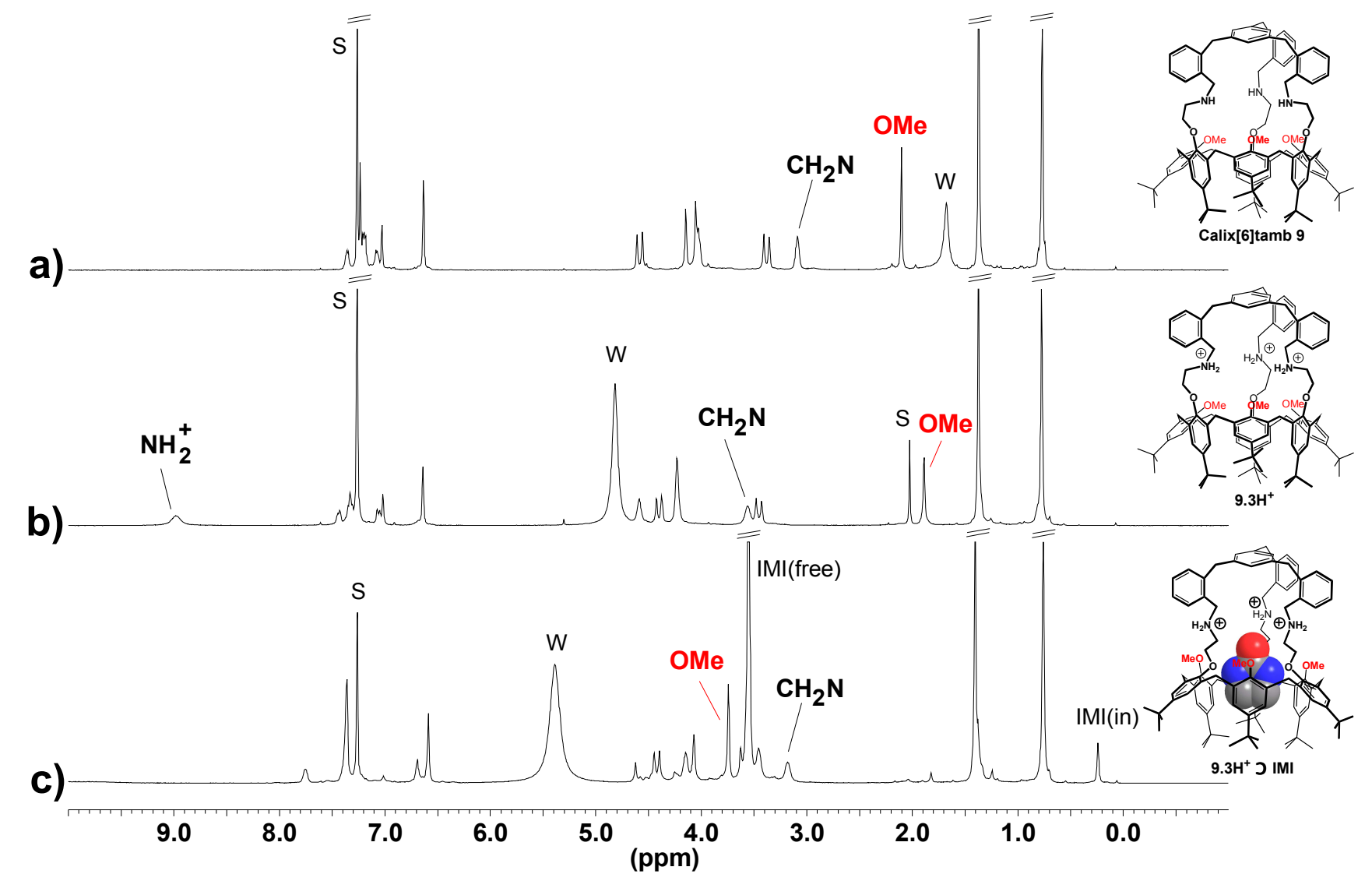

Figure S23. ${ }^{1} \mathrm{H}$ NMR spectra $\left(300 \mathrm{MHz}, \mathrm{CDCl}_{3}\right)$ of a) calix[6]tamb 9; b) fully protonated

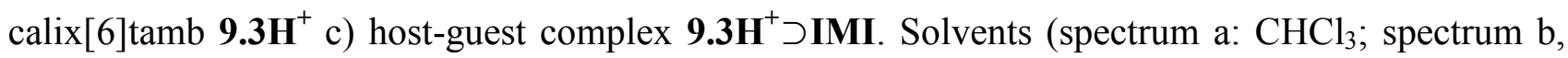
from the left: $\mathrm{CHCl}_{3}, \mathrm{CH}_{3} \mathrm{CN}$; spectrum c: $\mathrm{CHCl}_{3}$ ) and water have been labeled "S" and "W", respectively. 